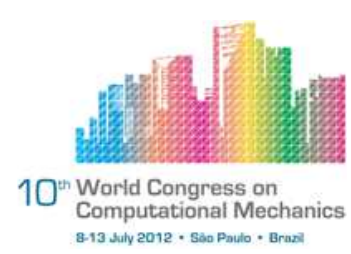

\title{
A STABLE GALERKIN REDUCED ORDER MODEL (ROM) FOR COMPRESSIBLE FLOW
}

\author{
I. Kalashnikova ${ }^{1}$, S. Arunajatesan ${ }^{2}$ \\ ${ }^{1}$ Numerical Analysis and Applications Department, Sandia National Laboratories \\ (ikalash@sandia.gov). \\ ${ }^{2}$ Aerosciences Department, Sandia National Laboratories.
}

\begin{abstract}
A method for constructing stable Proper Orthogonal Decomposition (POD)/Galerkin reduced order models (ROMs) for compressible flow is described. The proposed model reduction technique differs from the approach taken in many applications in that the Galerkin projection step is applied to the continuous system of partial differential equations (PDEs), rather than a semi-discrete representation of these equations. It is demonstrated that the inner product used to define the Galerkin projection is intimately tied to the stability of the resulting model. For linearized conservation laws such as the linearized compressible Euler and linearized compressible Navier-Stokes equations, a symmetry transformation leads to a stable formulation for the inner product. Preservation of stability for the discrete implementation of the Galerkin projection is made possible using piecewise-smooth finite element bases, and a weak enforcement of the boundary conditions. The stability and accuracy of the proposed model reduction approach is studied in the context of two test cases: the problem of an inviscid pressure pulse in a uniform base flow, and a viscous laminar cavity problem. For the second inherently non-linear test case, the non-linear dynamics of the flow are captured in the $P O D$ reduced basis modes, but not in the equations projected onto these modes, as the ROM equations are based on a local linearization of the full non-linear flow equations.
\end{abstract}

Keywords: Reduced order model (ROM); proper orthogonal decomposition (POD)/Galerkin projection; compressible Navier-Stokes equations; energy method; numerical stability.

\section{INTRODUCTION}

Many engineering problems that are of interest in present-day applications require the simulation of complex systems possessing many degrees of freedom (DOFs). One area that presents many tough challenges for scientific computing tools because of the large systems that arise is computational fluid dynamics (CFD). Despite improved algorithms and increasingly sophisticated computational tools, "high-fidelity" CFD models for three-dimensional (3D) unsteady flows at high Reynolds and Mach numbers are often too computationally expensive to be used in a design or analysis setting. 
In recent years, reduced order modeling has proven to be a powerful tool that can enable real or near-real time analysis in areas that have traditionally relied on high-fidelity modeling approaches. The basic idea of model reduction is to replace a large system associated with a high-fidelity simulation by a system that is much smaller, but that is nonetheless capable of capturing, at least approximately, the essential dynamics of the original system. Examples of reduced order model (ROM) approaches proposed within the past decade, each with its own inherent strengths, are the reduced basis method [15, 24, 25], balanced truncation $[27,28]$, balanced proper orthogonal decomposition (POD) [29], moment-matching [30], and goal-oriented ROMs [16]. The attractiveness of ROMs in predictive settings has prompted improvements in model-reduction methodologies for applications such as flow controller design [17, 21, 42], shape optimization [22], and aeroelastic stability analysis [18, 23]. It has also motivated the development of approaches for adapting pre-computed ROMs to changes in physical and/or modeling parameters [17, 21, 18, 23, 19, 20].

In any application, it is important for a ROM to preserve certain crucial properties of the original system. This is especially the case if the ROM's intended use is in a predictive setting. Many model reduction techniques in fluid mechanics and other applications are derived from the proper orthogonal decomposition(POD)/Galerkin projection approach. In this approach, a reduced basis is computed by performing a singular value decomposition (SVD) on a matrix containing as its columns a small number of snapshots extracted from a high-fidelity simulation. The set of governing partial differential equations (PDEs), or a semi-discrete representation of these equations, is then projected onto these reduced basis (POD) modes. The result of this procedure is a dynamical system of ordinary differential equations (ODEs), from which the ROM solution is obtained.

The ROM dynamical system may be viewed as an alternative discretization of the governing PDEs. As such, it is desired to possess the fundamental numerical properties required of any numerical scheme, namely stability, consistency and convergence. For a compressible flow POD/Galerkin model, general results regarding any of these properties are lacking [16]. Stability in particular can be an issue. A ROM constructed for the equations of compressible flow using the classical POD/Galerkin approach might be stable for a given number of modes, but unstable for other choices of basis size $[16,1,2,3,8]$. This leads to obvious practical limitations of the model: the ROM solution can blow up in finite time.

The aim of the current work is to present a model reduction approach based on Galerkin projection that is guaranteed to result in a stable ROM for the equations of compressible flow. At the heart of this stable formulation is the idea that a ROM must be constructed to retain a proper energy balance $[31,32,28]$. Mathematically, the energy is expressed as an inner product. In [1, 2, 3, 8], it was demonstrated by the first author, Kalashnikova, et al. that the stability of the Galerkin projection step of a ROM is closely tied to the stability of the resulting model. In these earlier works, attention was focussed on the linearized compressible Euler equations. An energy stability analysis carried out for Galerkin methods applied to these equations resulted in the construction of an inner product that guarantees certain stability bounds satisfied by the ROM. ROMs developed using this approach were guaranteed, by construction, to remain stable not only for the POD basis, but for any choice of reduced basis.

In the present work, the earlier analysis $[1,2,3,8]$ is extended to the linearized 
compressible Navier-Stokes equations. It is demonstrated that the key ingredient to a stable Galerkin ROM for this equation set is once again a proper choice of inner product for the Galerkin projection step of the model reduction. The appropriate inner product for this equation set is derived, and a stability-preserving implementation of the proposed ROM is developed. As a first step towards a stable and efficient model reduction approach for non-linear compressible flow problems, the viability of a ROM constructed for the full non-linear compressible Navier-Stokes equations based on a local linearization of these equations is studied. In this approach, the non-linear dynamics of the flow are captured in the POD reduced basis modes, but not in the equations projected onto these modes. The method is hence similar in flavor to the method of localized linear reductions [34], but unlike other non-linear model reduction approaches based on non-linear equations (gappy POD method [33], "best points" interpolation method (BPIM) [36], Gauss-Newton with Approximated Tensors (GNAT) method [35]).

The remainder of this paper is organized as follows. The equations of viscous compressible flow are given in Section 2. The POD/Galerkin approach to model reduction is reviewed in Section 3. A method for constructing a Galerkin ROM for the linearized compressible Navier-Stokes that is guaranteed to satisfy some a priori stability bounds is described in Section 4. In Section 5, the performance of the proposed model reduction approach is evaluated on two test cases: the problem of an inviscid pressure pulse in a steady base flow, and a viscous laminar cavity problem. Comparisons are made to ROMs constructed using the classical Galerkin/POD approach, which lacks an a priori stability guarantee. Conclusions are offered in Section 6.

\section{EQUATIONS FOR COMPRESSIBLE FLOW}

The Navier-Stokes equations are the fundamental PDEs for describing viscous fluid flow. In 3D, the compressible form of these equations can be written as [13]:

$$
\begin{array}{ll}
\rho \frac{D u_{1}}{d t} & =-\frac{\partial p}{\partial x_{1}}+\sum_{j=1}^{3} \frac{\partial}{\partial x_{j}}\left\{\mu\left(\frac{\partial u_{1}}{\partial x_{j}}+\frac{\partial u_{j}}{\partial x_{1}}\right)+\lambda \delta_{1 j} \nabla \cdot \mathbf{u}\right\}, \\
\rho \frac{D u_{2}}{d t} & =-\frac{\partial p}{\partial x_{2}}+\sum_{j=1}^{3} \frac{\partial}{\partial x_{j}}\left\{\mu\left(\frac{\partial u_{2}}{\partial x_{j}}+\frac{\partial u_{j}}{\partial x_{2}}\right)+\lambda \delta_{2 j} \nabla \cdot \mathbf{u}\right\}, \\
\rho \frac{D u_{3}}{d t} & =-\frac{\partial p}{\partial x_{3}}+\sum_{j=1}^{3} \frac{\partial}{\partial x_{j}}\left\{\mu\left(\frac{\partial u_{3}}{\partial x_{j}}+\frac{\partial u_{j}}{\partial x_{3}}\right)+\lambda \delta_{3 j} \nabla \cdot \mathbf{u}\right\}, \\
\rho C_{v} \frac{D T}{d t} & =-p \nabla \cdot \mathbf{u}+\sum_{i=1}^{3} \frac{\partial}{\partial x_{i}}\left(\kappa \frac{\partial T}{\partial x_{i}}\right), \\
\frac{D \rho}{\partial t} & =-\rho \nabla \cdot \mathbf{u},
\end{array}
$$

where

$$
\frac{D}{d t} \equiv \frac{\partial}{\partial t}+\mathbf{u} \cdot \nabla
$$

In (1), $\mathbf{u}^{T} \equiv\left(u_{1}, u_{2}, u_{3}\right)$ is the fluid velocity vector, $T$ is the fluid temperature, $p$ is the fluid pressure, and $\rho$ is the fluid density. Typically the system (1) is closed by relating the fluid pressure to the fluid density and temperature through an equation of state, e.g., the ideal gas law. The symbols $\mu$ and $\lambda$ are the so-called Lamé constants, assumed to satisfy Stokes' hypothesis:

$$
\lambda+\frac{2}{3} \mu=0
$$


The symbol $\kappa$ is the thermal diffusivity, and the symbol $C_{v}$ is the specific heat at constant volume, assumed herein to be constant.

An important dimensionless parameter associated with (1) is the Reynolds number, denoted by $R e$ and defined as:

$$
R e \equiv \frac{\rho_{0} U_{0} L}{\mu_{0}},
$$

where $\rho_{0}, \mu_{0}$ and $U_{0}$ are reference values for the density, viscosity and velocity respectively, and $L$ is a reference length scale for the domain of the problem considered. In the limit $R e \rightarrow \infty$ (or the case when $\mu=\lambda=\kappa=0$ ), the equations (1) reduce to the (inviscid) compressible Euler equations, a quasi-linear hyperbolic system. For finite values of $R e$, the system is known as incompletely parabolic [12].

When $\mu, \lambda, \kappa \neq 0$, that is, when viscosity is prevalent in the flow, appropriate solidwall boundary conditions for the equations (1) are the

$$
\begin{array}{lcl}
\text { no-slip boundary condition: } & \mathbf{u}=\mathbf{0}, & \text { on } \partial \Omega_{W}, \\
\text { adiabatic wall boundary condition: } & \nabla T \cdot \mathbf{n}=0, & \text { on } \partial \Omega_{W},
\end{array}
$$

where $\partial \Omega_{W}$ denotes a solid-wall boundary of the domain $\Omega$ on which the equations (1) are posed, and $\mathbf{n}^{T} \equiv\left(n_{1}, \quad n_{2}, \quad n_{3}\right)$ denotes the unit normal to $\partial \Omega_{W}$.

When $\mu=\lambda=\kappa=0$, that is, when the flow is assumed to be inviscid, so that the equations (1) reduce to the compressible Euler equations, an appropriate solid-wall boundary is the

$$
\text { no-penetration boundary condition: } \mathbf{u} \cdot \mathbf{n}=0 \text {, on } \partial \Omega_{W} \text {. }
$$

In numerical approximations to (1), the computational domain $\Omega$ on which these equations are solved is by construction finite, in contrast to the infinite physical space on which an initial boundary value problem (IBVP) for (1) may be defined. It is often desirable to prescribe boundary conditions on the artificial far-field boundary of the computational domain, denoted by $\partial \Omega_{F}$. An appropriate far-field boundary condition is one that will suppress the reflection of waves from the outer computational domain boundary. This can be accomplished by setting the components of $\mathbf{q}^{\prime}$ corresponding to characteristic waves traveling into $\Omega$ to zero $[1,8]$.

\subsection{Linearized Compressible Navier-Stokes Equations}

The model reduction approach presented in the present work is based on a local linearization of the full non-linear compressible Navier-Stokes equations (1). In general, the linearized compressible Navier-Stokes equations are appropriate when a compressible fluid system can be described by viscous, small-amplitude perturbations about a steady-state mean (or base) flow. To derive the linearized counterpart of the system (1), the state vector

$\mathbf{q}^{T} \equiv\left(u_{1}, \quad u_{2}, \quad u_{3}, \quad T, \quad \rho\right) \in \mathbb{R}^{5}$ is decomposed into two parts: a steady mean $\overline{\mathbf{q}}(\mathbf{x})$ and an unsteady time-varying fluctuation $\mathbf{q}^{\prime}(\mathbf{x}, t)$. Mathematically,

$$
\mathbf{q}(\mathbf{x}, t) \equiv \overline{\mathbf{q}}(\mathbf{x})+\mathbf{q}^{\prime}(\mathbf{x}, t),
$$

where $\mathbf{x}^{T} \equiv\left(x_{1}, x_{2}, x_{3}\right)$ is the position vector. Linearizing the full equations (1) around the steady mean (or base) state $\overline{\mathbf{q}}$, the following equations for the fluctuation $\mathbf{q}^{\prime}$ are obtained ${ }^{1}$

\footnotetext{
${ }^{1}$ From this point forward, the so-called Eisenstein notation (implied summation on repeated indices) is employed.
} 
$[12,13]:$

$$
\mathbf{q}_{, t}^{\prime}+\mathbf{A}_{i} \mathbf{q}_{, i}^{\prime}-\left[\mathbf{K}_{i j} \mathbf{q}_{, j}^{\prime}\right]_{, i}=\mathbf{0}
$$

where

$$
\begin{gathered}
\mathbf{A}_{1} \equiv\left(\begin{array}{ccccc}
\bar{u}_{1} & 0 & 0 & R & \frac{R \bar{T}}{\bar{\rho}} \\
0 & \bar{u}_{1} & 0 & 0 & 0 \\
0 & 0 & \bar{u}_{1} & 0 & 0 \\
\bar{T}(\gamma-1) & 0 & 0 & \bar{u}_{1} & 0 \\
\bar{\rho} & 0 & 0 & 0 & \bar{u}_{1}
\end{array}\right), \quad \mathbf{A}_{2} \equiv\left(\begin{array}{cccccc}
\bar{u}_{2} & 0 & 0 & 0 & 0 \\
0 & \bar{u}_{2} & 0 & R & \frac{R \bar{T}}{\bar{\rho}} \\
0 & 0 & \bar{u}_{2} & 0 & 0 \\
0 & \bar{T}(\gamma-1) & 0 & \bar{u}_{2} & 0 \\
0 & \bar{\rho} & 0 & 0 & \bar{u}_{2}
\end{array}\right), \\
\mathbf{A}_{3} \equiv\left(\begin{array}{ccccc}
\bar{u}_{3} & 0 & 0 & 0 & 0 \\
0 & \bar{u}_{3} & 0 & 0 & 0 \\
0 & 0 & \bar{u}_{3} & R & \frac{R \bar{T}}{\bar{\rho}} \\
0 & 0 & \bar{T}(\gamma-1) & \bar{u}_{3} & 0 \\
0 & 0 & \bar{\rho} & 0 & \bar{u}_{3}
\end{array}\right),
\end{gathered}
$$

and

$$
\begin{aligned}
& \mathbf{K}_{11} \equiv \frac{1}{\bar{\rho}}\left(\begin{array}{ccccc}
2 \mu+\lambda & 0 & 0 & 0 & 0 \\
0 & \mu & 0 & 0 & 0 \\
0 & 0 & \mu & 0 & 0 \\
0 & 0 & 0 & \frac{(\gamma-1) \kappa}{R} & 0 \\
0 & 0 & 0 & 0 & 0
\end{array}\right), \quad \mathbf{K}_{12} \equiv \frac{1}{\bar{\rho}}\left(\begin{array}{ccccc}
0 & \lambda & 0 & 0 & 0 \\
\mu & 0 & 0 & 0 & 0 \\
0 & 0 & 0 & 0 & 0 \\
0 & 0 & 0 & 0 & 0 \\
0 & 0 & 0 & 0 & 0
\end{array}\right), \\
& \mathbf{K}_{13} \equiv \frac{1}{\bar{\rho}}\left(\begin{array}{ccccc}
0 & 0 & \lambda & 0 & 0 \\
0 & 0 & 0 & 0 & 0 \\
\mu & 0 & 0 & 0 & 0 \\
0 & 0 & 0 & 0 & 0 \\
0 & 0 & 0 & 0 & 0
\end{array}\right), \quad \mathbf{K}_{21} \equiv \frac{1}{\bar{\rho}}\left(\begin{array}{ccccc}
0 & \mu & 0 & 0 & 0 \\
\lambda & 0 & 0 & 0 & 0 \\
0 & 0 & 0 & 0 & 0 \\
0 & 0 & 0 & 0 & 0 \\
0 & 0 & 0 & 0 & 0
\end{array}\right) \\
& \mathbf{K}_{22} \equiv \frac{1}{\bar{\rho}}\left(\begin{array}{ccccc}
\mu & 0 & 0 & 0 & 0 \\
0 & 2 \mu+\lambda & 0 & 0 & 0 \\
0 & 0 & \mu & 0 & 0 \\
0 & 0 & 0 & \frac{(\gamma-1) \kappa}{R} & 0 \\
0 & 0 & 0 & 0 & 0
\end{array}\right), \quad \mathbf{K}_{23} \equiv \frac{1}{\bar{\rho}}\left(\begin{array}{ccccc}
0 & 0 & 0 & 0 & 0 \\
0 & 0 & \lambda & 0 & 0 \\
0 & \mu & 0 & 0 & 0 \\
0 & 0 & 0 & 0 & 0 \\
0 & 0 & 0 & 0 & 0
\end{array}\right) \text {, } \\
& \mathbf{K}_{31} \equiv \frac{1}{\bar{\rho}}\left(\begin{array}{ccccc}
0 & 0 & \mu & 0 & 0 \\
0 & 0 & 0 & 0 & 0 \\
\lambda & 0 & 0 & 0 & 0 \\
0 & 0 & 0 & 0 & 0 \\
0 & 0 & 0 & 0 & 0
\end{array}\right), \quad \mathbf{K}_{32} \equiv \frac{1}{\bar{\rho}}\left(\begin{array}{ccccc}
0 & 0 & 0 & 0 & 0 \\
0 & 0 & \mu & 0 & 0 \\
0 & \lambda & 0 & 0 & 0 \\
0 & 0 & 0 & 0 & 0 \\
0 & 0 & 0 & 0 & 0
\end{array}\right), \\
& \mathbf{K}_{33} \equiv \frac{1}{\bar{\rho}}\left(\begin{array}{ccccc}
\mu & 0 & 0 & 0 & 0 \\
0 & \mu & 0 & 0 & 0 \\
0 & 0 & 2 \mu+\lambda & 0 & 0 \\
0 & 0 & 0 & \frac{(\gamma-1) \kappa}{R} & 0 \\
0 & 0 & 0 & 0 & 0
\end{array}\right) \text {. }
\end{aligned}
$$

In deriving (8), the ideal gas law

$$
p=\rho R T,
$$

where $R$ is the universal gas constant, has been employed. In addition, the identity $C_{v}=\frac{R}{\gamma-1}$, where $\gamma$ is the ratio of specific heats, has been invoked.

The equations (8) are known as the linearized compressible Navier-Stokes equations. When $\mathbf{K}_{i j} \equiv \mathbf{0}$ for $i, j=1,2,3$, (8) reduces to the linearized compressible Euler system. 


\subsection{Symmetrized Linearized Compressible Navier-Stokes Equations}

A key property of the incompletely parabolic system (8), one that is critical to the formulation of a stable Galerkin ROM for these equations, is that it is symmetrizable [12, 11]. In other words, there exists a symmetric positive definite matrix $\mathbf{H} \equiv \mathbf{H}(\mathbf{q})$ so that the following two properties hold:

1. The convective flux matrices

$$
\mathbf{A}^{S} \equiv \mathbf{H A}_{i}, \quad \text { for } i=1,2,3
$$

are all symmetric.

2. The following augmented viscosity matrix

$$
\mathbf{K}^{S} \equiv\left(\begin{array}{lll}
\mathbf{H K}_{11} & \mathbf{H K}_{12} & \mathbf{H K}_{13} \\
\mathbf{H K}_{21} & \mathbf{H K}_{22} & \mathbf{H K}_{23} \\
\mathbf{H K}_{31} & \mathbf{H K}_{32} & \mathbf{H K}_{33}
\end{array}\right),
$$

is symmetric positive semi-definite $\left(\mathbf{v}^{T} \mathbf{K}^{S} \mathbf{v} \geq 0\right.$ for all vectors $\left.\mathbf{v}\right)$.

To demonstrate that the system (8) is symmetrizable, a symmetrizer matrix $\mathbf{H}$ such that the two properties listed above are satisfied is exhibited. Consider the following matrix:

$$
\mathbf{H} \equiv\left(\begin{array}{ccccc}
\bar{\rho} & 0 & 0 & 0 & 0 \\
0 & \bar{\rho} & 0 & 0 & 0 \\
0 & 0 & \rho & 0 & 0 \\
0 & 0 & 0 & \frac{\bar{\rho} R}{\bar{T}(\gamma-1)} & 0 \\
0 & 0 & 0 & 0 & \frac{R \bar{T}}{\bar{\rho}}
\end{array}\right) .
$$

Let $\mathbf{A}_{i}^{S} \equiv \mathbf{H A}_{i}$ and $\mathbf{K}_{i j}^{S} \equiv \mathbf{H K}_{i j}$ denote the symmetrized convective and viscous flux matrices respectively, for $i, j=1,2,3$. Some linear algebra reveals that, with $\mathbf{H}$ defined by (18), these matrices are given by:

$$
\begin{aligned}
& \mathbf{A}_{1}^{S} \equiv\left(\begin{array}{ccccc}
\bar{\rho} \bar{u}_{1} & 0 & 0 & \bar{\rho} R & R \bar{T} \\
0 & \bar{\rho} \bar{u}_{1} & 0 & 0 & 0 \\
0 & 0 & \bar{\rho} \bar{u}_{1} & 0 & 0 \\
\bar{\rho} R & 0 & 0 & \frac{R \bar{\rho} \bar{u}_{1}}{\bar{T}(\gamma-1)} & 0 \\
R \bar{T} & 0 & 0 & 0 & \frac{R \bar{T} \bar{u}_{1}}{\bar{\rho}}
\end{array}\right), \quad \mathbf{A}_{2}^{S} \equiv\left(\begin{array}{ccccc}
\bar{\rho} \bar{u}_{2} & 0 & 0 & 0 & 0 \\
0 & \bar{\rho} \bar{u}_{2} & 0 & \bar{\rho} R & R \bar{T} \\
0 & 0 & \bar{\rho} \bar{u}_{2} & 0 & 0 \\
0 & \bar{\rho} R & 0 & \frac{R \rho \bar{u} \bar{u}_{2}}{\bar{T}(\gamma-1)} & 0 \\
0 & R \bar{T} & 0 & 0 & \frac{R \bar{T} \bar{u}_{2}}{\bar{\rho}}
\end{array}\right), \\
& \mathbf{A}_{3}^{S} \equiv\left(\begin{array}{ccccc}
\bar{\rho} \bar{u}_{3} & 0 & 0 & 0 & 0 \\
0 & \bar{\rho} \bar{u}_{3} & 0 & 0 & 0 \\
0 & 0 & \bar{\rho} \bar{u}_{3} & \bar{\rho} R & R \bar{T} \\
0 & 0 & \bar{\rho} R & \frac{R \bar{\rho} \bar{u}_{3}}{T(\gamma-1)} & 0 \\
0 & 0 & R \bar{T} & 0 & \frac{R \bar{T} \bar{u}_{3}}{\bar{\rho}}
\end{array}\right)
\end{aligned}
$$

and

$$
\mathbf{K}_{11}^{S} \equiv\left(\begin{array}{ccccc}
2 \mu+\lambda & 0 & 0 & 0 & 0 \\
0 & \mu & 0 & 0 & 0 \\
0 & 0 & \mu & 0 & 0 \\
0 & 0 & 0 & \frac{\kappa}{T} & 0 \\
0 & 0 & 0 & 0 & 0
\end{array}\right), \quad \mathbf{K}_{12}^{S} \equiv\left(\begin{array}{ccccc}
0 & \lambda & 0 & 0 & 0 \\
\mu & 0 & 0 & 0 & 0 \\
0 & 0 & 0 & 0 & 0 \\
0 & 0 & 0 & 0 & 0 \\
0 & 0 & 0 & 0 & 0
\end{array}\right), \quad \mathbf{K}_{13}^{S} \equiv\left(\begin{array}{ccccc}
0 & 0 & \lambda & 0 & 0 \\
0 & 0 & 0 & 0 & 0 \\
\mu & 0 & 0 & 0 & 0 \\
0 & 0 & 0 & 0 & 0 \\
0 & 0 & 0 & 0 & 0
\end{array}\right),
$$




$$
\begin{aligned}
& \mathbf{K}_{21}^{S} \equiv\left(\begin{array}{ccccc}
0 & \mu & 0 & 0 & 0 \\
\lambda & 0 & 0 & 0 & 0 \\
0 & 0 & 0 & 0 & 0 \\
0 & 0 & 0 & 0 & 0 \\
0 & 0 & 0 & 0 & 0
\end{array}\right), \quad \mathbf{K}_{22}^{S} \equiv\left(\begin{array}{ccccc}
\mu & 0 & 0 & 0 & 0 \\
0 & 2 \mu+\lambda & 0 & 0 & 0 \\
0 & 0 & \mu & 0 & 0 \\
0 & 0 & 0 & \frac{\kappa}{T} & 0 \\
0 & 0 & 0 & 0 & 0
\end{array}\right), \quad \mathbf{K}_{23}^{S} \equiv\left(\begin{array}{ccccc}
0 & 0 & 0 & 0 & 0 \\
0 & 0 & \lambda & 0 & 0 \\
0 & \mu & 0 & 0 & 0 \\
0 & 0 & 0 & 0 & 0 \\
0 & 0 & 0 & 0 & 0
\end{array}\right), \\
& \mathbf{K}_{31}^{S} \equiv\left(\begin{array}{ccccc}
0 & 0 & \mu & 0 & 0 \\
0 & 0 & 0 & 0 & 0 \\
\lambda & 0 & 0 & 0 & 0 \\
0 & 0 & 0 & 0 & 0 \\
0 & 0 & 0 & 0 & 0
\end{array}\right), \quad \mathbf{K}_{32}^{S} \equiv\left(\begin{array}{ccccc}
0 & 0 & 0 & 0 & 0 \\
0 & 0 & \mu & 0 & 0 \\
0 & \lambda & 0 & 0 & 0 \\
0 & 0 & 0 & 0 & 0 \\
0 & 0 & 0 & 0 & 0
\end{array}\right), \quad \mathbf{K}_{33}^{S} \equiv\left(\begin{array}{ccccc}
\mu & 0 & 0 & 0 & 0 \\
0 & \mu & 0 & 0 & 0 \\
0 & 0 & 2 \mu+\lambda & 0 & 0 \\
0 & 0 & 0 & \frac{\kappa}{T} & 0 \\
0 & 0 & 0 & 0 & 0
\end{array}\right) .
\end{aligned}
$$

It is clear that the convective flux matrices $\mathbf{A}_{i}^{S}$ (19) are symmetric, for $i=1,2,3$. The reader may verify that the augmented symmetrized viscous flux matrix $\mathbf{K}^{S}$ (17), with submatrices given by (20)-(22), is symmetric positive semi-definite.

It is noted that any matrix $\mathbf{H}$ that symmetrizes the viscous equations (8) is also a valid symmetrizer for the inviscid counterpart of these equations ((8) with $\mathbf{K}_{i j} \equiv \mathbf{0}$ for $i, j=$ $1,2,3)$. The converse is not in general true, however. That is, a symmetrizer for the linearized compressible Euler equations is not necessarily a symmetrizer for the linearized compressible Navier-Stokes equations.

\section{THE POD/GALERKIN APPROACH TO MODEL REDUCTION}

Before describing the proposed model reduction approach, a brief overview of the POD/Galerkin method for reducing the order of a complex physical system governed by a general set of PDEs is provided. The approach consists of two steps, summarized in Sections 3.1 and 3.2 below.

\subsection{Calculation of a Reduced Basis via the Proper Orthogonal Decomposition (POD)}

The first step in the POD/Galerkin method for model reduction is the calculation of a reduced basis using the POD of an ensemble of realizations from a high-fidelity simulation. In effect, this step involves the transfer of kinematic information from a high-fidelity simulation to a relatively small number of modes. Discussed in detail in Lumley [14] and Holmes et al. [15], POD is a mathematical procedure that, given an ensemble of data, constructs a basis for that ensemble that is optimal in the sense that it describes more energy (on average) of the ensemble than any other linear basis of the same dimension $M$. In the present context, the ensemble $\left\{u^{k}(x): k=1, \ldots, K\right\}$ is a set of $K$ instantaneous snapshots of a high-fidelity numerical solution field. Mathematically, POD seeks an $M$-dimensional $(M<<K)$ subspace $\mathcal{H}^{M}(\Omega)$ spanned by the set $\left\{\phi_{i}\right\}$ such that the projection of the difference between the ensemble $u^{k}$ and its projection onto $\mathcal{H}^{M}(\Omega)$ is minimized on average. It is a well known result $[1,15,10,9]$ that the solution to this optimization problem reduces to the eigenvalue problem $\mathcal{R} \phi=\lambda \phi$ where $\mathcal{R} \equiv\left\langle u^{k} \otimes u^{k}\right\rangle$ is a self-adjoint and positive semi-definite operator. It can be shown $[15,14]$ that the set of $M$ eigenfunctions, or POD modes, $\left\{\phi_{i}: i=1,2, \ldots, M\right\}$ corresponding to the $M$ largest eigenvalues of $\mathcal{R}$ is precisely the set of $\left\{\phi_{i}\right\}$ that solves the aforementioned POD optimization problem. Given this basis, the numerical ROM solution 
$u_{M}$ can be represented as a linear combination of POD modes

$$
u_{M}(x, t)=\sum_{j=1}^{M} a_{j}(t) \phi_{j}(x),
$$

where the $a_{j}(t)$ are the so-called ROM coefficients (or modal amplitudes) to be solved for in the ROM.

\subsection{Galerkin Projection of Governing PDEs onto Reduced Basis Modes}

The second step in constructing a ROM involves projecting the governing system of PDEs onto the reduced basis $\left\{\phi_{i}\right\}$ in some appropriate inner product, denoted generically (for now) by $(\cdot, \cdot)$. In this step, the full-system dynamics are effectively translated to the implied dynamics of the POD modes. If the governing system of equations for the state variable vector $u$ has the form

$$
\frac{\partial u}{\partial t}=\mathcal{L} u+\mathcal{N}_{2}(u, u)+\mathcal{N}_{3}(u, u, u)
$$

where $\mathcal{L}$ is a linear differential operator, and $\mathcal{N}_{2}$ and $\mathcal{N}_{3}$ are (non-linear) quadratic and cubic operators respectively, then the Galerkin projection of (24) onto the POD mode $\phi_{j}$ for $j=$ $1,2, \ldots, M$ is

$$
\left(\phi_{j}, \frac{\partial u_{M}}{\partial t}\right)=\left(\phi_{j}, \mathcal{L} u_{M}\right)+\left(\phi_{j}, \mathcal{N}_{2}\left(u_{M}, u_{M}\right)\right)+\left(\phi_{j}, \mathcal{N}_{3}\left(u_{M}, u_{M}, u_{M}\right)\right) .
$$

Substituting the POD decomposition of $u_{M}$ (23) into (25) and applying the orthonormality property of the basis functions $\phi_{i}$ in the inner product $(\cdot, \cdot)$ gives a set of time-dependent ODEs in the modal amplitudes:

$$
\begin{aligned}
\frac{d a}{d t} \equiv \dot{a}_{j} & =\sum_{l=1}^{M} a_{l}\left(\phi_{j}, \mathcal{L}\left(\phi_{l}\right)\right)+\sum_{l=1}^{M} \sum_{m=1}^{M} a_{l} a_{m}\left(\phi_{j}, \mathcal{N}_{2}\left(\phi_{l}, \phi_{m}\right)\right) \\
& +\sum_{l=1}^{M} \sum_{m=1}^{M} \sum_{n=1}^{M} a_{l} a_{m} a_{n}\left(\phi_{j}, \mathcal{N}_{3}\left(\phi_{l}, \phi_{m}, \phi_{n}\right)\right),
\end{aligned}
$$

for $j=1,2, \ldots, M$. This ROM dynamical system accurately describes the flow dynamics of the full system of PDEs for some limited set of flow conditions.

\subsection{Continuous vs. Discrete Projection}

As suggested by equations (25)-(26) and as will become apparent in Section 4, the approach proposed in the present work, termed the "continuous projection" approach, is based on a Galerkin projection of the continuous governing PDEs onto the reduced basis modes. Many POD/Galerkin applications are based on the so-called "discrete projection", in which the semi-discrete representation of the governing equations is projected, and numerical analysis proceeds from the perspective of a dynamical system of ODEs. The continuous projection approach has the advantage that the ROM solution behavior can be examined using methods that have been used for numerical analysis of spectral $[6,5]$ and finite volume $[4,7]$ approximations to PDEs. This makes possible an a priori stability analysis using the energy method (Theorem 4.1). Note that, in the continuous projection approach, boundary condition terms present in the discretized equation set are not in general inherited by the ROM, and therefore may need to be implemented in the ROM (Section 4.2). 


\section{A STABILITY-PRESERVING POD/GALERKIN MODEL REDUCTION APPROACH FOR LINEARIZED COMPRESSIBLE FLOW}

The discussion of the Galerkin projection step of the model reduction in Section 3.2 has assumed a generic inner product $(\cdot, \cdot)$. In the classical POD/Galerkin method, this inner product is taken to be the $L^{2}(\Omega)$ inner product. For the incompressible Navier-Stokes equations, this choice of inner product is appropriate, and will give rise to a stable POD/Galerkin ROM. The reason for this is that, for this equation set, the solution vector is the velocity vector $\mathbf{u}$, and $\|\mathbf{u}\|_{L^{2}(\Omega)}$ is a measure of the global kinetic energy in the domain $\Omega$. The $L^{2}(\Omega)$ inner product is therefore physically sensible for these equations: the POD modes optimally represent the kinetic energy present in the ensemble from which they are generated.

The stability of the Galerkin projection of a PDE system in a given inner product can be studied using the energy method, reviewed briefly here. Consider the following Cauchy problem for a scalar PDE

$$
\begin{array}{ll}
\frac{\partial u}{\partial t} & =\mathcal{L} u, \quad \mathbf{x} \in \mathbb{R}^{n}, \quad t \geq 0, \\
u(\mathbf{x}, 0) & =f(\mathbf{x}),
\end{array}
$$

where $\mathcal{L}$ is a linear spatial differential operator. The basic idea of the energy method [12, 37] is to define the energy of a solution $u$ to (27) in an inner product $(\cdot, \cdot)_{E}$ with corresponding norm $\|\cdot\|_{E}$ as

$$
E \equiv\|u\|_{E}
$$

and attempt to derive a bound of the form

$$
\frac{1}{2} \frac{d}{d t}\|u\|_{E} \leq \alpha\|u\|_{E}, \quad \alpha \in \mathbb{R} .
$$

If such a bound can be shown, the operator $\mathcal{L}$ is semi-bounded with respect to the inner product $(\cdot, \cdot)_{E}$, and the Cauchy problem (27) is well-posed. Consequently, a Galerkin approximation to $u$, denoted by $u_{N}$, will satisfy the energy estimate

$$
\frac{1}{2} \frac{d}{d t}\left\|u_{N}\right\|_{E} \leq \alpha\left\|u_{N}\right\|_{E}, \quad \alpha \in \mathbb{R}
$$

so that, by Gronwall's lemma,

$$
\left\|u_{N}(\mathbf{x}, t)\right\|_{E} \leq e^{\alpha t}\left\|u_{N}(\mathbf{x}, 0)\right\|_{E}, \quad \forall t \geq 0 .
$$

The practical implication of the result (31) is that if the energy inner product $(\cdot, \cdot)_{E}$ is selected for the Galerkin approximation, the resulting numerical solution will be bounded in a way that is consistent with the behavior of the exact solutions of the original PDE, i.e., it will be stable.

It turns out that the $L^{2}(\Omega)$ inner product does not correspond to an energy integral for equations of compressible flow, such as the ones considered herein $[1,2,3,8]$. The practical implication of this fact is that if the $L^{2}(\Omega)$ inner product is selected as the inner product defining the projection, the resulting ROM will not satisfy the energy conservation relation implied by the governing equations, and may exhibit an instability that is inconsistent with the physics inherent in the governing continuous PDEs [16]. An alternate inner product, in particular, one that is an energy inner product for the equations of compressible flow, is sought. 


\subsection{Stability-Preserving Symmetry Inner Product for the Galerkin Projection Step}

As will be proven in Section 4.3, the energy inner product for the system (8) is the so-called "symmetry", or $(\mathbf{H}, \Omega)$-, inner product, defined by:

$$
\left(\mathbf{v}_{1}, \mathbf{v}_{2}\right)_{(\mathbf{H}, \Omega)} \equiv \int_{\Omega} \mathbf{v}_{1}^{T} \mathbf{H} \mathbf{v}_{2} d \Omega
$$

for $\mathbf{v}_{1}, \mathbf{v}_{2}, \mathbf{v} \in \mathbb{R}^{5}$. The matrix $\mathbf{H}$ is precisely the matrix that symmetrizes the linearized compressible Navier-Stokes system (8). Projecting the (continuous) system of PDEs onto the $k^{\text {th }}$ reduced basis mode for $k=1, \ldots, M$ in the $(\mathbf{H}, \Omega)$-inner product gives:

$$
\int_{\Omega} \boldsymbol{\phi}_{k}^{T} \mathbf{H} \mathbf{q}_{t}^{\prime} d \Omega+\int_{\Omega} \boldsymbol{\phi}_{k}^{T} \mathbf{A}_{i}^{S} \mathbf{q}_{, i}^{\prime} d \Omega-\int_{\Omega} \boldsymbol{\phi}_{k}^{T}\left[\mathbf{K}_{i j}^{S} \mathbf{q}_{, j}^{\prime}\right]_{, i} d \Omega=0
$$

where $M$ is the total number of reduced basis modes. The norm corresponding to the inner product (32), referred to as the $(\mathbf{H}, \Omega)$-norm, is given by:

$$
\|\mathbf{v}\|_{(\mathbf{H}, \Omega)} \equiv \sqrt{(\mathbf{v}, \mathbf{v})_{(\mathbf{H}, \Omega)}} .
$$

(34) defines a valid norm, as the symmetrizer matrix $\mathbf{H}$ is symmetric positive definite.

\subsection{Stability-Preserving Weak Implementation of Boundary Conditions}

As mentioned in Section 3.3, a Galerkin ROM based on the continuous projection approach does not necessarily inherit boundary condition terms present in the discretized equation set from which the ROM is constructed. An efficient implementation of the boundary conditions for a Galerkin ROM is through a weak formulation. For illustration purposes, attention is restricted to a solid-wall boundary of the domain $\Omega$, on which the no-slip and adiabatic wall boundary conditions (5) are imposed. First, the system of PDEs (8) is projected onto the $k^{t h}$ reduced basis mode in the $(\mathbf{H}, \Omega)$-inner product, as done in (33). Next, the viscous terms in (33) are integrated by parts:

$$
\int_{\Omega} \boldsymbol{\phi}_{k}^{T}\left(\mathbf{K}_{i j}^{S} \mathbf{q}_{, i}^{\prime}\right)_{, j} d \Omega=-\int_{\Omega} \boldsymbol{\phi}_{k, j}^{T} \mathbf{K}_{i j}^{S} \mathbf{q}_{, i}^{\prime} d \Omega+\underbrace{\int_{\partial \Omega} \boldsymbol{\phi}_{k}^{T} \mathbf{K}_{i j}^{S} \mathbf{q}_{, i}^{\prime} n_{j} d \Gamma}_{\equiv I_{k}^{W}} .
$$

Finally, the vector specifying the boundary condition is inserted into the boundary integral over $\partial \Omega$ that arises. Some simple linear algebra reveals that

$\mathbf{K}_{i j}^{S} \mathbf{q}_{, i}^{\prime} n_{j}=\left(\begin{array}{c}{\left[(2 \mu+\lambda) u_{1,1}^{\prime}+\mu\left(u_{2,2}+u_{3,3}\right)\right] n_{1}+\left(\lambda u_{2,1}^{\prime}+\mu u_{1,2}^{\prime}\right) n_{2}+\left(\lambda u_{3,1}^{\prime}+\mu u_{1,3}^{\prime}\right) n_{3}} \\ \left(\mu u_{2,1}^{\prime}+\lambda u_{1,2}^{\prime}\right) n_{1}+\left[\mu\left(u_{1,1}^{\prime}+u_{3,3}^{\prime}\right)+(2 \mu+\lambda) u_{2,2}^{\prime}\right] n_{2}+\left(\lambda u_{3,2}^{\prime}+\mu u_{2,3}^{\prime}\right) n_{3} \\ \left(\mu u_{3,1}^{\prime}+\lambda u_{1,3}^{\prime}\right) n_{1}+\left(\mu u_{3,2}^{\prime}+\lambda u_{2,3}^{\prime}\right) n_{2}+\left[\mu\left(u_{1,1}^{\prime}+u_{2,2}^{\prime}\right)+(2 \mu+\lambda) u_{3,3}^{\prime}\right] n_{3} \\ \frac{\kappa}{T} \nabla T^{\prime} \cdot \mathbf{n} \\ 0\end{array}\right)$.

Substituting the adiabatic wall boundary condition (5) and assuming the reduced basis modes $\phi_{k}$ satisfy the no-slip condition on $\partial \Omega_{W}$, it follows that

$$
\phi_{k}^{T} \mathbf{K}_{i j}^{S} \mathbf{q}_{, i}^{\prime} n_{j}=0 .
$$


Substituting (37) into (35), the following Galerkin-projected ROM formulation with boundary conditions is obtained:

$$
\int_{\Omega} \boldsymbol{\phi}_{k}^{T} \mathbf{H} \mathbf{q}_{t}^{\prime} d \Omega+\int_{\Omega} \boldsymbol{\phi}_{k}^{T} \mathbf{A}_{i}^{S} \mathbf{q}_{, i}^{\prime} d \Omega+\int_{\Omega} \boldsymbol{\phi}_{k, i}^{T} \mathbf{K}_{i j}^{S} \mathbf{q}_{, j}^{\prime} d \Omega=0 .
$$

The ROM matrix problem is obtained by inserting the modal decomposition $\mathbf{q}^{\prime} \approx \mathbf{q}_{M}^{\prime}=$ $\sum_{i=1}^{M} a_{i}(t) \phi_{i}(\mathbf{x})$ into (38) (Section 5.1).

\subsection{Proof of Energy-Stability of the Symmetry Inner Product}

The present section provides a proof of the energy-stability of the Galerkin projection step of the model reduction procedure in the $(\mathbf{H}, \Omega)$-inner product, assuming a weak implementation of the boundary conditions (5) following the procedure outlined in Section 4.2. The far-field boundary condition on $\partial \Omega_{F}$ is not considered as it is stable by construction (proven in $[8])$.

Theorem 4.1. Consider a bounded domain $\Omega \subset \mathbb{R}^{3}$ with Lipshitz-continuous boundary solidwall boundary $\partial \Omega_{W}$. Assume the modes $\phi_{j}$ and the base flow satisfy a no-slip condition on $\partial \Omega_{W}$ (the first condition in (5)). Then the Galerkin projection of (8) with boundary conditions (5) is "energy-stable" in the symmetry inner product (32), with energy estimate

$$
\left\|\mathbf{q}^{\prime}(\cdot, T)\right\|_{(\mathbf{H}, \Omega)} \leq \exp \left\{\frac{1}{2} \beta T\right\}\left\|\mathbf{q}^{\prime}(\cdot, 0)\right\|_{(\mathbf{H}, \Omega)},
$$

where $\beta$ is an upper bound on the eigenvalues of the matrix ${ }^{2}$

$$
\mathbf{B} \equiv \mathbf{H}^{-T / 2} \frac{\partial\left(\mathbf{H A}_{i}\right)}{\partial x_{i}} \mathbf{H}^{-1 / 2} .
$$

Proof. By Definition 2.11 in [37] (repeated in the Appendix), to show stability of the Galerkin projection of $(8)$ in the $(\mathbf{H}, \Omega)$-inner product, it is sufficient to show that the energy associated with this projection is semi-bounded in the associated $(\mathbf{H}, \Omega)$-norm. Defining the energy as

$$
E \equiv\left\|\mathbf{q}^{\prime}\right\|_{(\mathbf{H}, \Omega)}
$$

the following bound on $\frac{d E}{d t}$ is obtained:

$$
\begin{aligned}
\frac{1}{2} \frac{d}{d t}\left\|\mathbf{q}^{\prime}\right\|_{(\mathbf{H}, \Omega)} & =\frac{1}{2} \frac{d}{d t} \int_{\Omega}\left[\mathbf{q}^{\prime}\right]^{T} \mathbf{H} \mathbf{q} d \Omega \\
& =\int_{\Omega}\left[\mathbf{q}^{\prime}\right]^{T} \mathbf{H} \mathbf{q}_{t}^{\prime} d \Omega \\
& =-\int_{\Omega}\left[\mathbf{q}^{\prime}\right]^{T} \mathbf{H} \mathbf{A}_{i} \mathbf{q}_{, i} d \Omega+\int_{\Omega}\left[\mathbf{q}^{\prime}\right]^{T} \mathbf{H}\left[\mathbf{K}_{i j} \mathbf{q}_{i}^{\prime}\right]_{, j} d \Omega \\
& =-\frac{1}{2} \int_{\Omega} \frac{\partial}{\partial x_{i}}\left(\left[\mathbf{q}^{\prime}\right]^{T} \mathbf{H} \mathbf{A}_{i} \mathbf{q}^{\prime} d \Omega\right) d \Omega+\frac{1}{2} \int_{\Omega}\left[\mathbf{q}^{\prime}\right]^{T} \frac{\partial\left(\mathbf{H} \mathbf{A}_{i}\right)}{\partial x_{i}} \mathbf{q}^{\prime} d \Omega-\int_{\Omega}\left[\mathbf{q}_{, j}^{\prime}\right]^{T} \mathbf{H} \mathbf{K}_{i j} \mathbf{q}_{i}^{\prime} d \Omega \\
& +\int_{\partial \Omega}\left[\mathbf{q}^{\prime}\right]^{T} \mathbf{K}_{i j}^{S} \mathbf{q}_{i}^{\prime} n_{j} d \Gamma \\
& =-\frac{1}{2} \int_{\partial \Omega}\left[\mathbf{q}^{\prime}\right]^{T} \mathbf{A}_{i}^{S} n_{i} \mathbf{q}^{\prime} d \Gamma+\frac{1}{2} \int_{\Omega}\left[\mathbf{q}^{\prime}\right]^{T} \frac{\partial\left(\mathbf{H} \mathbf{A}_{i}\right)}{\partial x_{i}} \mathbf{q}^{\prime} d \Omega-\int_{\Omega}\left[\mathbf{q}_{, j}^{\prime}\right]^{T} \mathbf{H} \mathbf{K}_{i j} \mathbf{q}_{i}^{\prime} d \Omega \\
& +\int_{\partial \Omega}\left[\mathbf{q}^{\prime}\right]^{T} \mathbf{K}_{i j}^{S} \mathbf{q}_{i}^{\prime} n_{j} d \Gamma
\end{aligned}
$$

\footnotetext{
${ }^{2}$ The shorthand $\left(\mathbf{M}^{1 / 2}\right)^{T} \equiv \mathbf{M}^{T / 2}$ is employed, where $\mathbf{M}$ is a positive definite matrix and $\mathbf{M}^{1 / 2}$ is its square root factor, so that $\mathbf{M}=\mathbf{M}^{T / 2} \mathbf{M}^{1 / 2}$.
} 
In going from lines 3 to 4 of (42), the symmetry property of the $\mathbf{A}_{i}^{S}$ matrices has been employed. Assuming the base flow satisfies a no-slip boundary condition at the wall, so that $\overline{\mathbf{u}} \cdot \mathbf{n}=0$,

$$
\mathbf{A}_{i}^{S} n_{i} \mathbf{q}^{\prime}=\left(\begin{array}{ccccc}
0 & 0 & 0 & \bar{\rho} R n_{1} & R \bar{T} n_{1} \\
0 & 0 & 0 & \bar{\rho} R n_{2} & R \bar{T} n_{2} \\
0 & 0 & 0 & \bar{\rho} R n_{3} & R \bar{T} n_{3} \\
\bar{\rho} R n_{1} & \bar{\rho} R n_{2} & \bar{\rho} R n_{3} & 0 & 0 \\
R \bar{T} n_{1} & R \bar{T} n_{2} & R \bar{T} n_{3} & 0 & 0
\end{array}\right)\left(\begin{array}{c}
u_{1}^{\prime} \\
u_{2}^{\prime} \\
u_{3}^{\prime} \\
T^{\prime} \\
\rho^{\prime}
\end{array}\right)
$$

Assuming that the reduced basis modes satisfy the no-slip boundary condition, it follows that

$$
\left[\mathbf{q}^{\prime}\right]^{T} \mathbf{A}_{i}^{S} n_{i} \mathbf{q}^{\prime}=0
$$

on $\partial \Omega_{W}$. Substituting (44) and (37) into (42), and using the fact that $\mathbf{K}^{S}$ (17) is positive semi-definite, one has that:

$$
\frac{d}{d t}\left\|\mathbf{q}^{\prime}\right\|_{(\mathbf{H}, \Omega)} \leq \int_{\Omega}\left[\mathbf{q}^{\prime}\right]^{T} \frac{\partial\left(\mathbf{H A}_{i}\right)}{\partial x_{i}} \mathbf{q}^{\prime} d \Omega
$$

Applying Gronwall's lemma to (45) gives (40).

The energy estimate (40) establishes the semi-boundedness of the governing spatial differential operator defined in (8) in the $(\mathbf{H}, \Omega)$-inner product. A direct consequence of this result is that the Galerkin projection step using the symmetry inner product is guaranteed to produce a stable ROM, provided well-posed (stability-preserving) boundary conditions are prescribed.

It is noted that the proof of Theorem 4.1 holds for the linearized compressible Euler equations - that is, equations (8) with $\mu=\lambda=\kappa=0$. The symmetrization presented in this paper for the linearized compressible Navier-Stokes equations thus encompasses the viscous as well as inviscid case, and is hence more general that the original symmetrization approach developed for the linearized compressible Euler equations [1, 2, 3, 8]. For the inviscid variant of (8), care must be taken to implement the appropriate boundary conditions (no-penetration at a solid wall (6)) in a way that preserves the stability of the ROM. For a detailed discussion of a stability-preserving implementation of inviscid boundary conditions, the reader is referred to [8].

\section{NUMERICAL EXPERIMENTS}

The model reduction based on the POD method and Galerkin projection of the continuous linearized compressible Navier-Stokes equations in the symmetry inner product described in the previous sections is now evaluated on two test cases. Emphasis is placed on reproducing a given CFD solution for a single set of flow conditions in a stable and accurate fashion. This is viewed as an essential prerequisite for applying the method to more complex situations, and employing the ROM in a predictive setting. 


\subsection{Implementation of the ROM}

Prior to evaluating the ROM's performance, some key details of the implementation of the ROM are described. The ROM can be thought of as consisting of two stages: an "offline" construction stage, and an "online" solution stage.

In the offline stage of the model reduction, the ROM matrix problem is assembled. Substituting the modal decomposition $\mathbf{q}^{\prime} \approx \mathbf{q}_{M}^{\prime}=\sum_{m=1}^{M} a_{m}(t) \phi_{m}$ into (38) and employing the fact that the POD modes are by construction orthonormal in the $(\mathbf{H}, \Omega)$-inner product yields the following linear dynamical system

$$
\dot{\mathbf{a}}=\mathbf{C a},
$$

where

$$
C_{k m}=-\int_{\Omega} \boldsymbol{\phi}_{k}^{T} \mathbf{A}_{i}^{S} \boldsymbol{\phi}_{m, i} d \Omega-\int_{\Omega} \boldsymbol{\phi}_{k, i}^{T} \mathbf{K}_{i j}^{S} \boldsymbol{\phi}_{m, j} d \Omega,
$$

for $k, m=1, \ldots, M$, with $M$ denoting the number of reduced basis modes. The offline stage of the ROM is implemented in a $\mathrm{C}++$ code that uses distributed vector and matrix data structures and parallel eigensolvers from the Trilinos project [39]. Given a set of snapshots from a highfidelity simulation stored at the nodes of an underlying mesh, this code computes a POD basis by solving the POD eigenvalue problem described in Section 3.1 using eigensolvers from the Trilinos RBGen library. Within this code, the POD basis functions are interpolated using piece-wise linear finite element shape functions, and the continuous Galerkin projection of the governing equations onto these POD modes is performed. For the Galerkin projection step of the model reduction, a numerical quadrature operator that exactly integrates the relevant inner products (47) is constructed with the help of the libmesh finite element library [40].

Although computationally intensive, as it requires $\mathcal{O}(N)$ operations, where $N$ is the number of grid points in the high-fidelity simulation, the offline stage of the model reduction is performed only a single time to yield the $M \times M$ ROM dynamical system matrix $\mathrm{C}$ (47). Given this matrix, the online stage of the ROM begins. In this stage, the ROM dynamical system (46) is integrated in time using a classical time-integration scheme, such as the RungeKutta method. In contrast to the offline stage of the model reduction, the online stage is fast, cheap, and can be performed for on-the-spot computations, as it scales like $M$, where $M<<N$ is the small number of reduced basis modes.

The approach outlined above, described in more detail in [1], ensures that the stability results of the analysis in Section 4.3 are preserved by the discrete implementation. Moreover, it makes the ROM potentially compatible with any CFD code that can output a mesh and snapshot data stored at the nodes of this mesh.

For the results presented in the following two subsections, the high-fidelity fluid simulation data were generated using a Sandia in-house finite volume flow solver known as SIGMA CFD. This code is derived from LESLIE3D [41], a Large Eddy Simulations (LES) flow solver originally developed in the Computational Combustion Laboratory at the Georgia Institute of Technology. LESLIE3D solves the turbulent compressible flow equations using an explicit 2-4 MacCormack scheme. A hybrid scheme coupling the MacCormack scheme to flux difference splitting schemes is employed to capture shocks. For parallel execution, a Schwartz overlap algorithm with ghosting is used, with the MPI library providing routines for exchanging information in the overlap regions. For a detailed description of the schemes and models 
implemented within LESLIE3D, the reader is referred to [43, 44]. The SIGMA CFD flow solver is an extension of LESLIE3D that includes the following major modifications:

1. Extension of the time marching scheme options to include an implicit time marching scheme.

2. Addition of a higher order reconstruction based Roe flux scheme and a low dissipation skew symmetric scheme.

3. Addition of RANS turbulence models: $k-\epsilon, k-\omega$ and SST turbulence models in addition to the one-equation subgrid kinetic energy based LES models in LESLIE3D.

4. Implementation of SST-DES and Hybrid RANS-LES models.

5. Implementation of a wall layer model for LES applications.

In addition to these changes, various code cleanups, simplification of the data structures, generalization of the parallel decomposition, as well as porting to large clusters and thousands of processors have been carried out by the authors. Various boundary conditions necessary for the range of applications of interest to Sandia have also been implemented in SIGMA CFD.

The ROM dynamical systems (46) were advanced forward in time using a fourth order explicit Runge-Kutta scheme, implemented in MATLAB, with the same time step that was used in the CFD simulation used to generate the ROM.

\subsection{Inviscid Pressure Pulse}

The first test case is that of a two-dimensional (2D) inviscid acoustic pressure pulse in the following prismatic domain: $\Omega=(-1,1) \times(-1,1) \times(-1,-0.9) \in \mathbb{R}^{3}$. For this problem, the base flow was uniform, with the following values:

$$
\begin{gathered}
\bar{p}=101,325 \mathrm{~Pa} \\
\bar{T}=300 \mathrm{~K} \\
\bar{\rho}=\frac{\bar{p}}{R T}=1.17 \mathrm{~kg} / \mathrm{m}^{3} \\
\bar{u}_{1}=\bar{u}_{2}=\bar{u}_{3}=0.0 \mathrm{~m} / \mathrm{s} \\
\bar{c}=348.0 \mathrm{~m} / \mathrm{s} .
\end{gathered}
$$

In (48), $\bar{c} \equiv \sqrt{\gamma R \bar{T}}$ is the mean speed of sound. The problem was initialized with a pressure pulse in the middle of the domain:

$$
\begin{gathered}
p^{\prime}(\mathbf{x} ; 0)=141.9 e^{-10\left(x^{2}+y^{2}\right)}, \\
\rho^{\prime}(\mathbf{x} ; 0)=\frac{p^{\prime}(\mathbf{x} ; 0)}{R T}, \\
T^{\prime}(\mathbf{x} ; 0)=0, \\
u_{1}^{\prime}(\mathbf{x} ; 0)=u_{2}^{\prime}(\mathbf{x} ; 0)=u_{3}^{\prime}(\mathbf{x} ; 0)=0 .
\end{gathered}
$$

In terms of the mean values, the amplitude of the initial pressure pulse (49) was $0.001 \bar{\rho} \bar{c}^{2}$. The computational grid for this test case was composed of 3362 nodes, cast into 9600 tetrahedral finite elements within the ROM code. A no-penetration (slip wall) boundary condition (6) was imposed on all six sides of the domain. The high-fidelity CFD simulation from which 
the ROM was generated was performed until time $T=0.01$ seconds. During this simulation, the initial pressure pulse (49) reflected from the walls of the domain a number of times. Snapshots from this simulation were saved every $5 \times 10^{-5}$ seconds, to yield a total of 200 snapshots. These snapshots were employed to construct a 20 mode POD basis. Two different procedures were used to generate a fluid ROM for this problem: the POD/Galerkin method with the $(\mathbf{H}, \Omega)$ - (symmetry) inner product, and the POD/Galerkin method with the classical $L^{2}$ inner product. Using both the symmetry and the $L^{2}$ inner product, the POD modes captured essentially $100 \%$ of the snapshot energy.

Figure 1 shows a time history of the first two ROM modal amplitudes (circles) compared to the projection of the full CFD simulation onto the first two POD modes (solid lines) for the symmetry (a) and $L^{2}$ (b) ROMs. Mathematically, this figure compares as a function of time $t$ :

$$
a_{i}(t) \quad \text { vs. } \quad\left(\mathbf{q}_{\mathrm{CFD}}^{\prime}, \boldsymbol{\phi}_{i}\right)_{(\mathbf{H}, \Omega)}, \quad i=1,2,
$$

where $\mathrm{q}_{\mathrm{CFD}}^{\prime}$ is the high-fidelity CFD solution from which the ROMs were constructed. The reader may observe good agreement between the symmetry ROM and the full simulation (Figure 1(a)) for the time interval considered. In contrast, agreement between the $L^{2}$ ROM and the full simulation is reasonable only until approximately $t=0.005$ seconds (Figure 1(b)). The oscillations in the $L^{2}$ ROM modal amplitudes observed for $t>0.008$ seconds suggest the presence of an instability in the $L^{2}$ ROM.

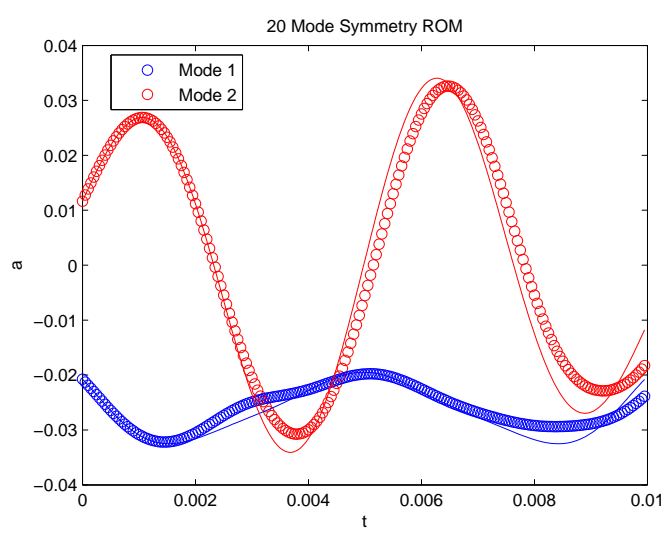

(a) 20 mode symmetry ROM

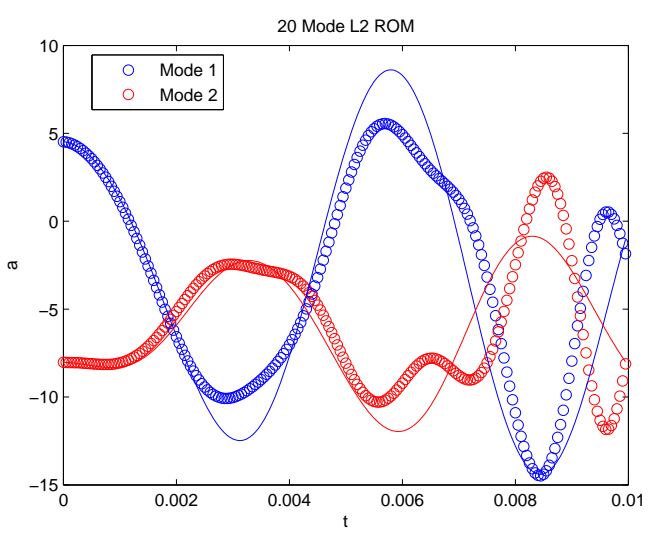

(b) 20 mode $L^{2}$ ROM

Figure 1. Time history of modal amplitudes for inviscid pressure pulse problem

Figures 2-4 compare the CFD pressure field (a) with the field reconstructed from the symmetry (b) and $L^{2}$ (c) ROM solutions at $z=-1$ and times $t=4.5 \times 10^{-4}, 2.95 \times 10^{-3}$ and $7.95 \times 10^{-3}$ seconds. At time $t=4.5 \times 10^{-4}$ seconds, both the symmetry and $L^{2}$ ROM solutions are in good agreement with the high-fidelity solution (Figure 2). At the later times, $t=2.95 \times 10^{-3}$ and $7.95 \times 10^{-3}$ seconds, there is a good qualitative agreement between the high-fidelity solution and the symmetry ROM solution (Figures 3-4(a), (b)). The same cannot be said of the $L^{2}$ ROM solution at these later times, however. It is apparent from Figure 4(c) that the $L^{2}$ ROM solution has blown up by $t=7.95 \times 10^{-3}$ seconds, which confirms the instability of the 20 mode $L^{2}$ ROM suggested in Figure 1. 


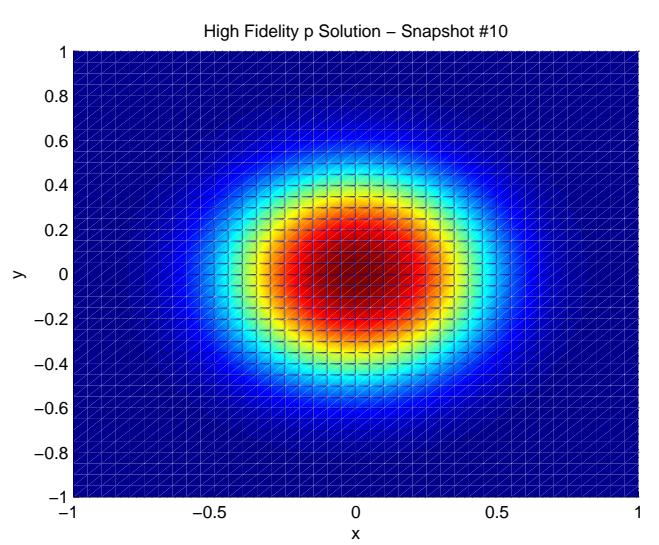

(a) $\mathrm{CFD}$

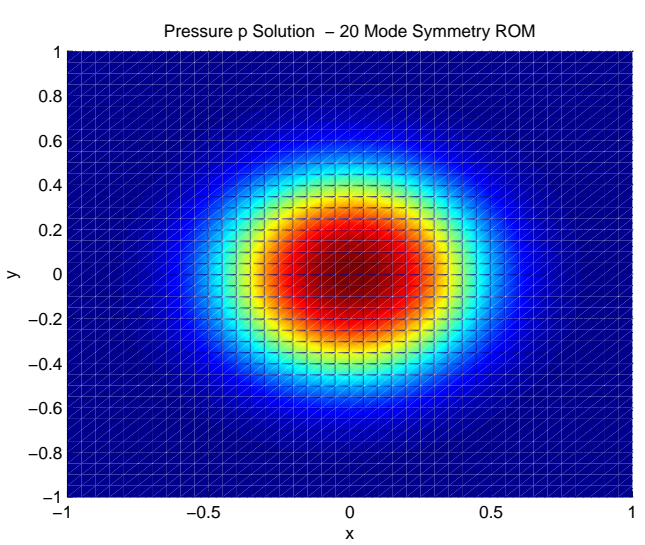

(b) 20 mode symmetry ROM

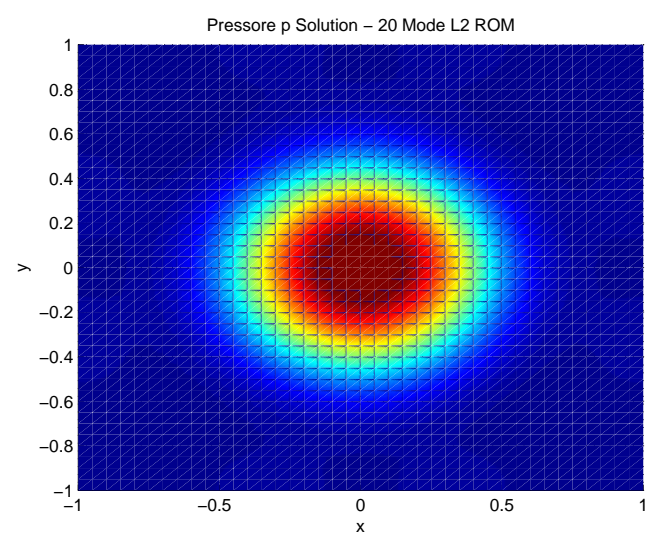

(c) 20 mode $L^{2} \mathrm{ROM}$

Figure 2. Pressure field at time $t=4.5 \times 10^{-4}$ seconds

\subsection{Viscous Laminar Cavity}

The second test case is that of a Mach 0.6 viscous laminar flow over a cavity in a $T$ shaped domain $\Omega=[(-2.9460,4.5857) \times(-0.4586,4.5857) \times(0,0.4586)] \backslash[(-2.9460,0) \times$ $(-0.4586,0) \times(0,0.4586)] \backslash[(0.9171,4.5857) \times(-0.4586,0) \times(0,0.4586)]$. The flow conditions correspond to case L2 in [38]. The free stream pressure was $25 \mathrm{~Pa}$, the free stream temperature was $300 \mathrm{~K}$, and the free stream velocity was $208.8 \mathrm{~m} / \mathrm{s}$. The free stream pressure was kept low to keep the Reynolds number of the flow low. The viscosity and thermal diffusivity coefficients were $\mu=1.846 \times 10^{-5} \mathrm{~kg} /(\mathrm{m} \cdot \mathrm{s})$ and $\kappa=2.587 \times 10^{-2} \mathrm{~m}^{2} / \mathrm{s}$ respectively. At the inflow boundary $x=-2.9460$, a value of the velocity and temperature that is above the free stream values was specified. The flow at the cavity walls $(x=-0.4586, x=0.9172$, $y=0, z=0, z=0.4586$ ) was assumed to be adiabatic and to satisfy a no-slip condition (5). The remaining outflow boundaries ( $y=4.5857, x=4.5857)$ were open, and a far-field boundary condition that suppresses the reflection of waves into the computational domain was implemented here. The simulation was started by initializing the flow in the cavity to have a zero velocity, free stream pressure, and temperature. The region above the cavity was initialized to free stream conditions and the flow was allowed to evolve. The snapshopts for constructing the ROM were collected once statistically stationary conditions were reached in 


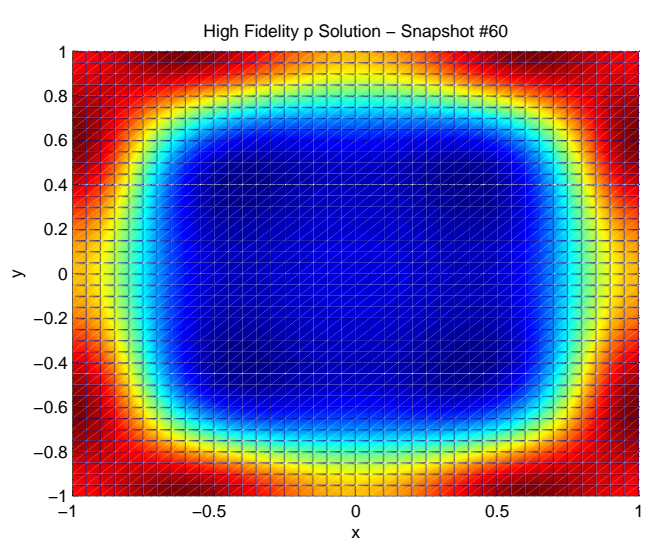

(a) $\mathrm{CFD}$

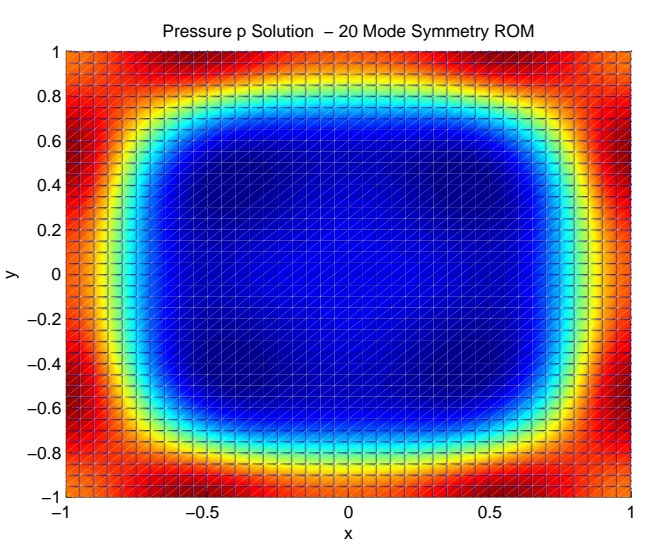

(b) 20 mode symmetry ROM

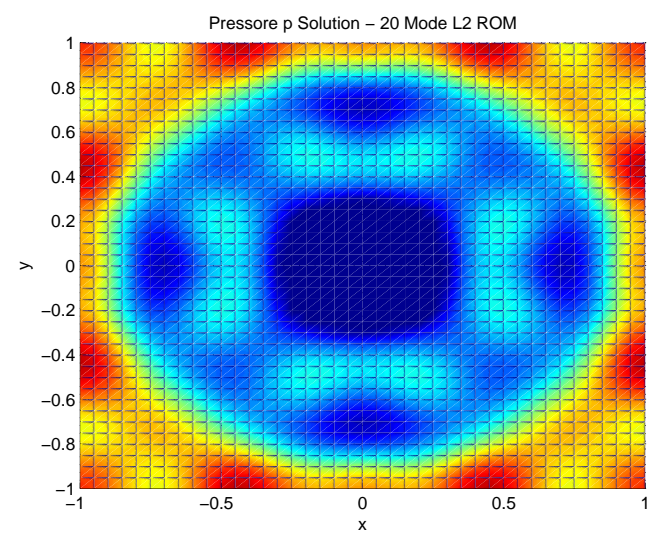

(c) 20 mode $L^{2} \mathrm{ROM}$

Figure 3. Pressure field at time $t=2.95 \times 10^{-3}$ seconds

the cavity. This was determined by examining the pressure fluctuations at several locations on the cavity walls.

In the high-fidelity viscous laminar cavity simulation, the full non-linear Navier-Stokes equations (1) in the laminar regime were solved. The discretized domain consisted of 343,408 nodes, which were cast as 1,020,000 tetrahedral finite elements within the ROM code. The high-fidelity CFD simulation was run until time $T=0.2$ seconds. Snapshots from this simulation were saved every $2 \times 10^{-3}$ seconds, to yield a total of 101 snapshots. From these snapshots, a 30 mode POD basis was constructed using the $L^{2}$ as well as the symmetry inner product (Figure 5). As for the inviscid pulse problem, these modes captured essentially $100 \%$ of the snapshot energy for both ROM approaches.

Unlike the inviscid pressure pulse problem considered in Section 5.2, the viscous laminar cavity problem is inherently non-linear, and does not possess a natural steady base flow component. In constructing the ROM, the base flow was taken to be the average of the snapshots. The full compressible Navier-Stokes equations (1) were linearized around this steady base flow to yield the linear system (8), and this system was subsequently projected onto the POD modes to yield the ROM dynamical system. In this approach, the non-linear dynamics of the flow (vortical structures, for the viscous laminar cavity flow problem) are captured in 


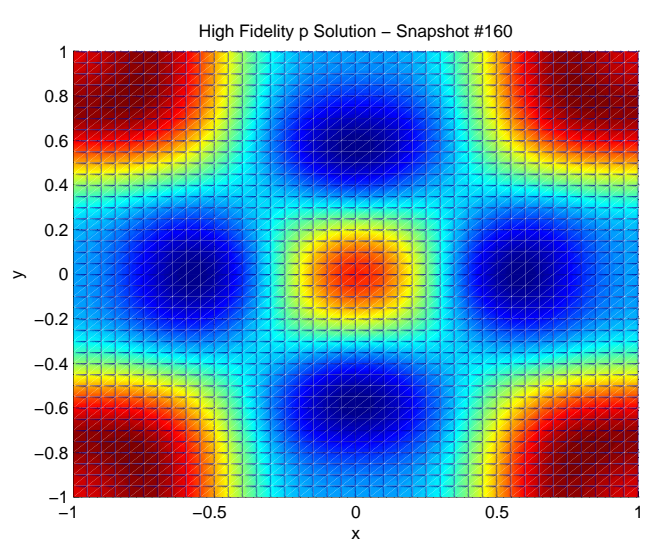

(a) $\mathrm{CFD}$

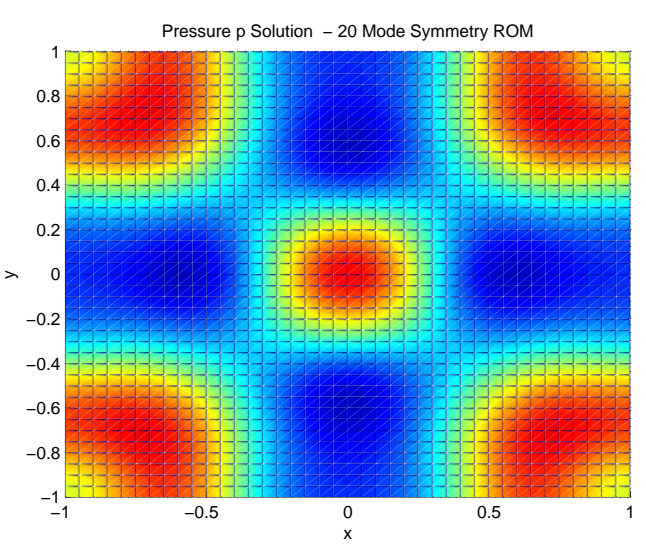

(b) 20 mode symmetry ROM

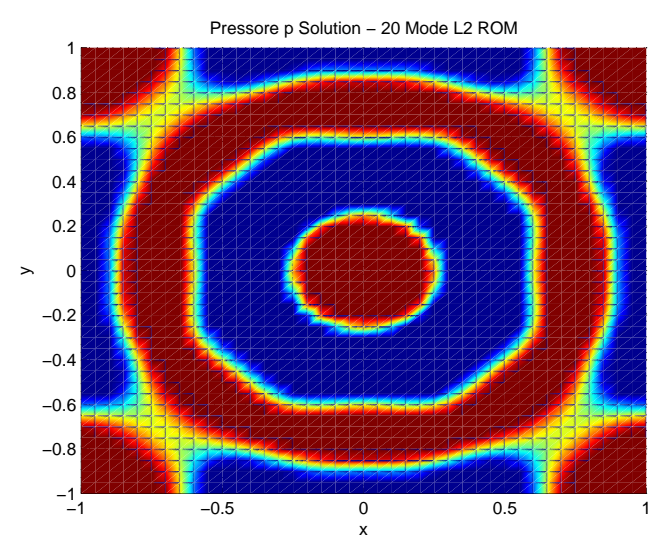

(c) 20 mode $L^{2} \mathrm{ROM}$

Figure 4. Pressure field at time $t=7.95 \times 10^{-3}$ seconds

the POD reduced basis modes (Figure 5), but not in the equations projected onto these reduced basis modes.

Since a ROM based on linearized equations cannot be expected to reproduce all the non-linear dynamics in the solution of an inherently non-linear problem such as the viscous laminar cavity problem, some discussion of what the ROM can and cannot be expected to capture is in order. The physics of the cavity flow are determined by the shear layer over the cavity. As the shear layer separates from the leading edge of the cavity, instabilities develop in the separated shear layer. These instabilities grow non-linearly to form vortices convecting down the shear layer. This process is an inviscid instability growth process and arises due to the non-linear terms in the convective part of the Navier-Stokes equations. Since the ROM is built using a linearized form of the Navier-Stokes equations, the ROM in its current form cannot be expected to capture accurately this process. Further downstream, the vortices impinge on the aft wall of the cavity giving rise to pressure waves that are propagated upstream through the free stream and the cavity. Depending upon the Reynolds number (and hence the free stream dynamic pressure), these waves can range from linear to non-linear. The linear waves (as is expected in this low Reynolds number case) should be accurately captured by the ROM. Since the pressure fluctuations on the cavity walls are due to a combination of 


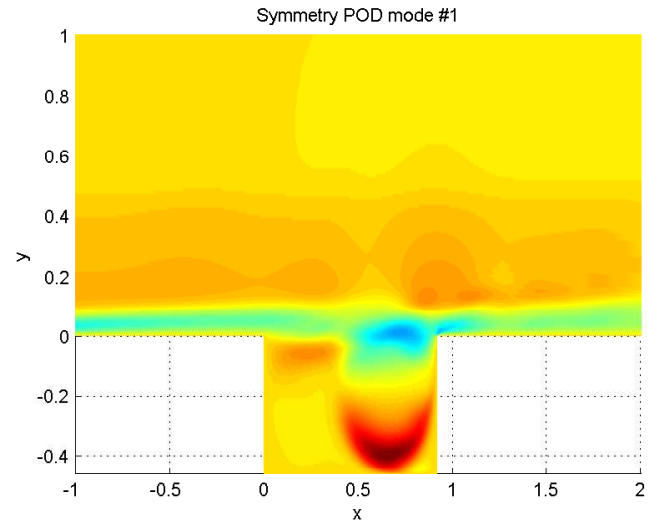

(a) Mode 1 (52.2\% energy)

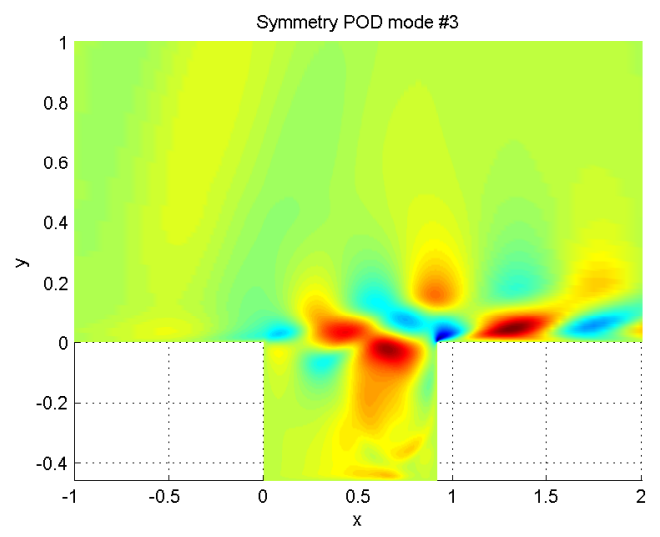

(c) Mode 3 (13.8\% energy)

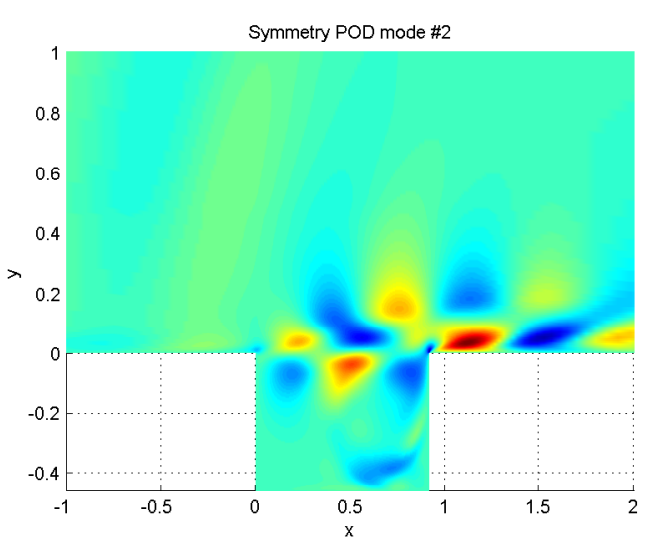

(b) Mode $2(15.5 \%$ energy)

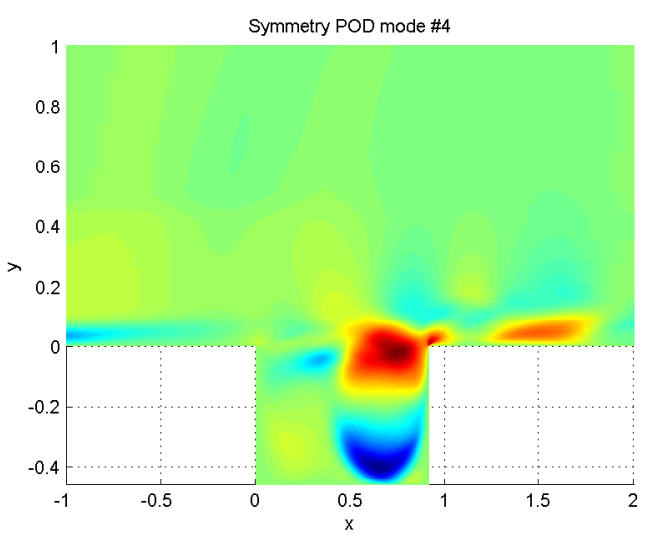

(d) Mode 4 (9.4\% energy)

Figure 5. First four most energetic POD modes computed in the symmetry inner product for the viscous laminar cavity problem

these waves and those generated by the shear layer vortices, it is expected that only the linear reflected waves will be captured by the ROM.

Figure 6 compares the CFD $u_{1}$ velocity field (a) with the field reconstructed from the symmetry (b) and $L^{2}$ (c) ROM solutions at time $t=0.024$ seconds and $z=0$. Figure 7 compares the CFD $u_{2}$ velocity field (a) with the field reconstructed form the symmetry (b) and $L^{2}$ (c) ROM solutions at a later time, $t=0.076$ seconds and $z=0$. The reader may observe that there is a reasonable qualitative agreement between both ROM solutions and the high-fidelity solution. The ROMs are not able to capture in full detail the inherently non-linear vortical structures present in the high-fidelity solution, but this is to be expected of a model based on linearized flow equations.

Figure 8 plots the real part of each eigenvalue of the $30 \times 30$ ROM dynamical system matrix $\mathbf{C}$ for the 30 mode symmetry and $L^{2}$ ROMs. The plot is an a posteriori check of the stability of each ROM. The ROM is stable if the maximum real part of the eigenvalues of the ROM system matrix is non-positive. Figure 8 confirms that the 30 mode symmetry ROM is stable. Although the 30 mode $L^{2}$ ROM is not observed to go unstable for this test case, it is clear from Figure 8 the stability of the $L^{2}$ ROM is not guaranteed, as its system matrix has eigenvalues with positive real parts. 


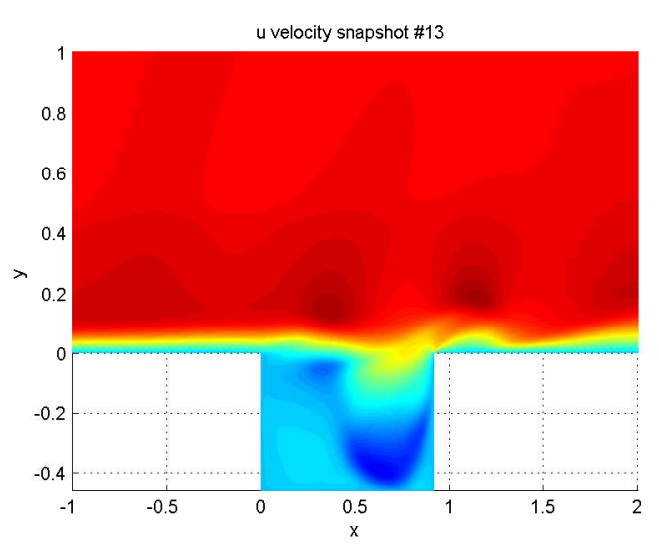

(a) $\mathrm{CFD}$

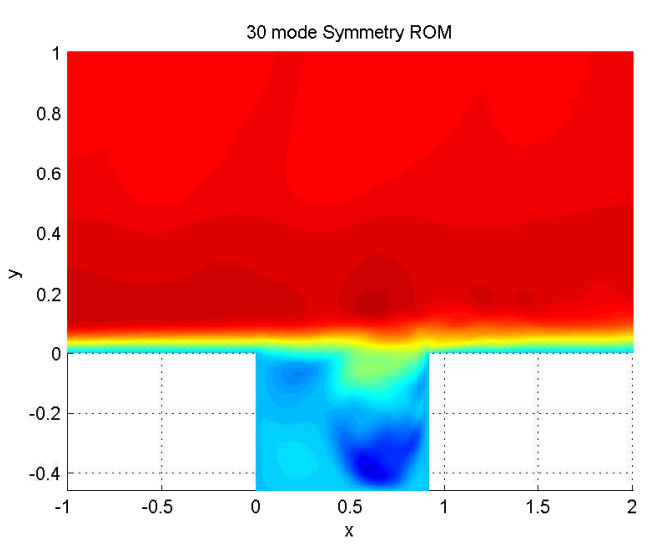

(b) 30 mode symmetry ROM

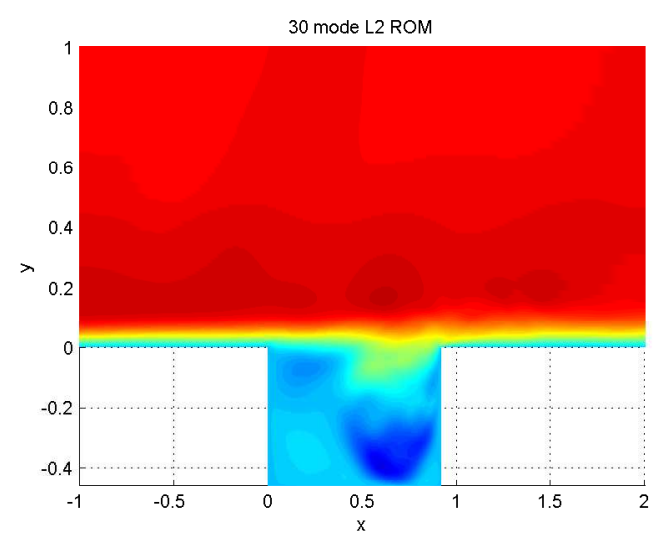

(c) 30 mode $L^{2} \mathrm{ROM}$

Figure 6. Velocity field $u_{1}$ at time $t=0.024$ seconds

\section{CONCLUSIONS}

A method for constructing stable reduced order models for compressible flow based on the POD/Galerkin approach has been developed and tested. Attention is focused on extending the earlier analysis and model reduction methodology $[1,2,3,8]$ to the linearized compressible Navier-Stokes equations, which were not considered in past work by the authors. For these equations, a symmetry inner product is defined such that the application of the Galerkin projection method in this inner product is guaranteed to produce stable ROMs for the governing equations. The proposed model reduction approach is evaluated on two test cases: the problem of an inviscid pressure pulse in a uniform base flow, and a laminar viscous cavity problem. For the first test case, there is a better qualitative agreement between the symmetry ROM solution and the high-fidelity solution, than the $L^{2}$ ROM solution and the high-fidelity solution. Moreover, the $L^{2} \mathrm{ROM}$ is observed to exhibit an instability, whereas the symmetry ROM remains stable for all times considered. The authors' objective in considering the second test case is to study the viability of a model reduction approach in which the non-linear dynamics of the flow are captured in the POD reduced basis modes, but not in the (locally linearized) equations projected onto these reduced basis modes. Results for this test case are encouraging: there is a reasonable qualitative agreement between the ROM solutions and the 


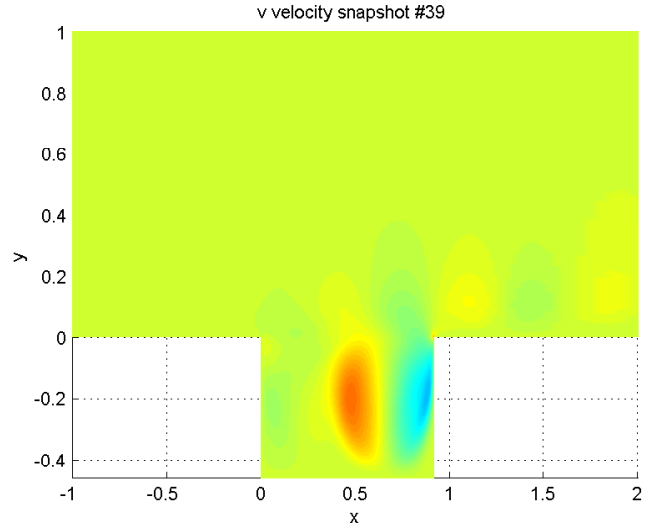

(a) CFD

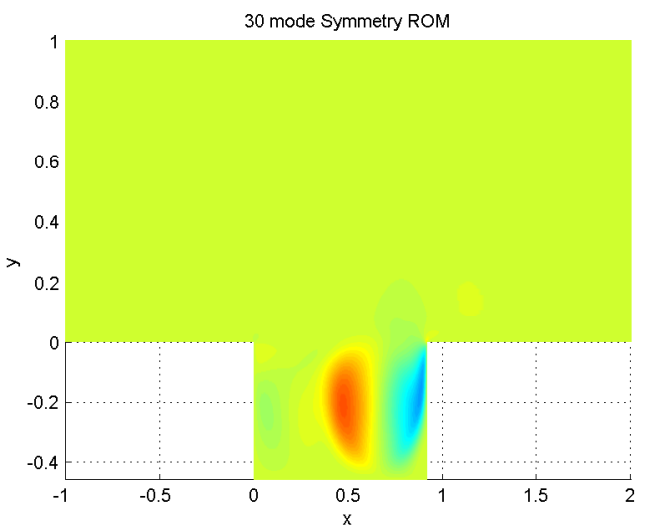

(b) 30 mode symmetry ROM

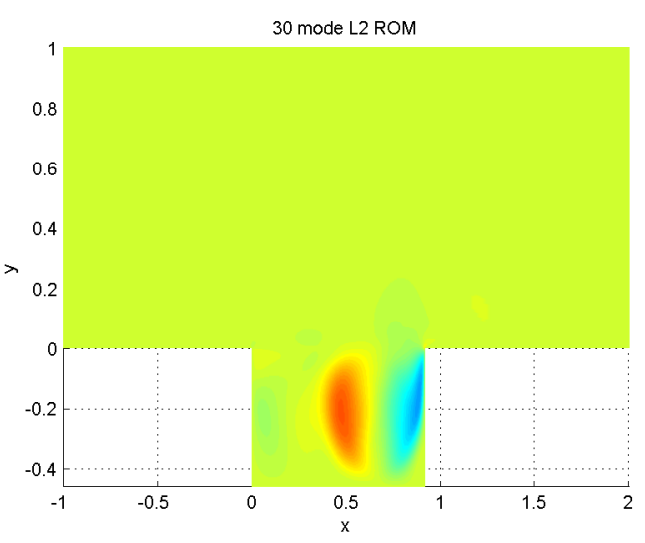

(c) 30 mode $L^{2} \mathrm{ROM}$

Figure 7. Velocity field $u_{2}$ at time $t=0.076$ seconds

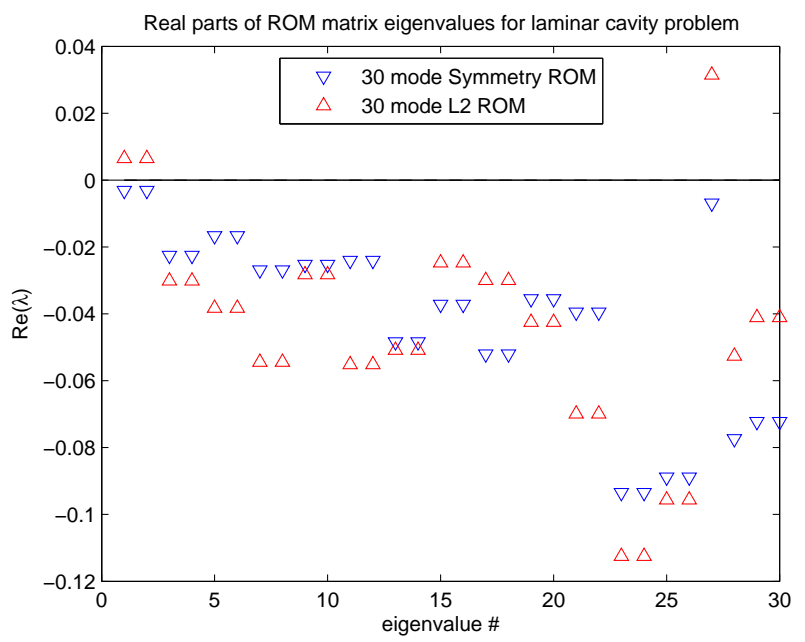

Figure 8. Real parts of eigenvalues of ROM system matrix $\mathrm{C}$ for viscous laminar cavity problem

high-fidelity solution. Future work will focus on incorporating into the ROM equations non- 
linear terms in a stability-preserving and computationally tractable way. Future work will also include a study of the predictive capabilities of the proposed ROM for long-time simulations, as well as an investigation of the ROM's robustness with respect to changes in the parameter space.

\section{APPENDIX}

Consider the following semi-discrete problem:

$$
\begin{aligned}
& \frac{d u_{j}}{d t}=Q u_{j}+F_{j}, \quad j=1,2, \ldots, N-1, \\
& \quad B_{h} u=g(t), \\
& u_{j}(0)=f_{j}, \quad j=1,2, \ldots, N,
\end{aligned}
$$

where $Q$ is a discretizing operator, $F_{j}$ and $f_{j}$ are the discretized version of $F$ and $f$ respectively, and $B_{h} u$ denotes the complete set of discretized boundary conditions. Let $\|\cdot\|_{h}$ be a discrete norm.

Definition 2.11 in [37]: The semi-discrete IBVP (51) is stable if there is a unique solution satisfying

$$
\|u(\cdot, t)\|_{h} \leq K e^{\beta t}\|f(\cdot)\| \|_{h},
$$

where $K$ and $\beta$ are constants independent of $f$ and $g$.

\section{Acknowledgements}

This research was funded by Sandia National Laboratories. Sandia is a multiprogram laboratory operated by Sandia Corporation, a Lockheed Martin Company for the United States Department of Energy's National Nuclear Security Administration under contract DE-AC0494AL85000.

The authors would like to thank Matthew Barone (Wind Power Technologies Department at Sandia National Laboratories) for many valuable discussions on model reduction and computational fluid dynamics.

\section{REFERENCES}

[1] M.F. Barone, I. Kalashnikova, D.J. Segalman, H. Thornquist. Stable Galerkin Reduced Order Models for Linearized Compressible Flow. J. Comput. Phys. 288 1932-1946 (2009).

[2] M.F. Barone, I. Kalashnikova, M.R. Brake, D.J. Segalman. Reduced Order Modeling of Fluid/Structure Interaction. Sandia National Laboratories Report, SAND No. 2009-7189. Sandia National Laboratories, Albuquerque, NM (2009).

[3] M.F. Barone, D.J. Segalman, H.K. Thornquist, I. Kalashnikova. Galerkin Reduced Order Models for Compressible Flow with Structural Interaction, AIAA 46th Aerospace Science Meeting and Exhibit, AIAA 2008-0612, Reno, NV (2008). 
[4] M. Gerritsen, P. Olsson. Designing an Efficient Solution Strategy for Fluid Flows: 1. A Stable High Order Finite Difference Scheme and Sharp Shock Resolution for the Euler Equations. J. Comput. Phys. 129 245-262 (1996).

[5] D. Funaro, D. Gottlieb. Convergence results for Pseudospectral Approximations of Hyperbolic Systems by a Penalty-Type Boundary Treatment, Math. of Comput. 57(196) 585-596 (1991).

[6] J.S. Hesthaven, D. Gottlieb. A Stable Method for the Compressible Navier-Stokes Equations: I. Open Boundary Conditions, SIAM J. Sci. Comput., 17(3) 579-612 (1996).

[7] A. Harten. On the symmetric form of systems of conservations laws with entropy. J. Comput. Phys. 49 151-164 (1983).

[8] I. Kalashnikova, M.F. Barone. On the Stability and Convergence of a Galerkin Reduced Order Model (ROM) of Compressible Flow with Solid Wall and Far-Field Boundary Treatment. Int. J. Numer. Meth. Engng. 83 1345-1375 (2010).

[9] M. Rathinam, L.R. Petzold. A New Look at Proper Orthogonal Decomposition, SIAM J. Numer. Anal. 41(5) 1893-1925 (2003).

[10] K. Kunisch, S. Volkwein. Galerkin Proper Orthogonal Decomposition for a General Equation in Fluid Dynamics, SIAM J. Numer. Anal., 40(2) 492-515 (2002).

[11] J.S. Hesthaven, D. Gottlieb. A stable penalty method for the compressible Navier-Stokes equations: I. Open boundary conditions. SIAM J. Sci. Comput. 17(3) 579-612 (1996).

[12] B. Gustafsson, A. Sundstrom. Incompletely parabolic problems in fluid dynamics. SIAM J. Appl. Math. 35(2) 343-357 (1978).

[13] L. Tourrette. Artificial boundary conditioners for the linearized Navier-Stokes equations. J. Comput. Phys. 137 1-37 (1997).

[14] J.L. Lumley. Stochastic Tools in Turbulence. New York, NY: Academic Press, 1971.

[15] P. Holmes, J.L. Lumley, G. Berkooz. Turbulence, Coherent Structures, Dynamical Systems and Symmetry, New York, NY: Cambridge University Press, 1996.

[16] T. Bui-Thanh, K. Willcox, O. Ghattas, and B. van Bloemen Waanders. Goal-oriented, model-constrained optimization for reduction of large-scale systems. J. Comp. Phys., 224 880-896 (2007).

[17] J.A. Taylor. Dynamics of Large Scale Structures in Turbulent Shear Layers. Ph.D. thesis, Clarkson University (2001).

[18] T. Lieu, C. Farhat. Aerodynamic parameter adaptation of CFD-based reduced-order models. 45th Aerospace Sciences Meeting and Exhibit, AIAA Paper 2007-328 (2007).

[19] D. Amsallem, C. Farhat. An interpolation method for adapting reduced-order models. AIAA J. 46(7) 1803-1813 (2008). 
[20] D. Amsallem, J. Cortial, K. Carlberg, C. Farhat. A method for interpolating on manifolds structural dynamics reduced-order models. Int. J. Numer. Meth. Engng. 80 1241-1258 (2009).

[21] J.A. Taylor, M.N. Glauser. Towards practical flow sensing and control via POD and LSE based low-dimensional tools. J. Fluids Engng. 126(3) 337-345 (2003).

[22] A. LeGresley, J.J. Alonso. Investigation of non-linear projection for POD based reduced order models for aerodynamics. 39th AIAA Aeroscpace Sciences Meeting \& Exhibit, AIAA Paper 2001-0926 (2001).

[23] T. Lieu, C. Farhat, M. Lesoinne. Reduced-order fluid/structure modeling of complete aircraft confirguration. Comput. Methods Appl. Mech. Engng. 195 5730-5742 (2006).

[24] L. Sirovich. Turbulence and the dynamics of coherent structures, Part III: Dynamics and scaling. Quarterly of Applied Mathematics 45(3) 583-590 (1987).

[25] N. Aubry, P. Holmes, J. Lumley, E. Stone. The dynamics of coherent structures in the wall region of a turbulent boundary layer. J. Fluid. Mech. 192 115-173 (1988).

[26] K. Veroy, A.T. Patera. Certified real-time solution of the parametrized steady incompressible Navier-Stokes equations: rigorous reduced-basis a posteriori error bounds. Int. J. Numer. Meth. Fluids 47 773-788 (2005).

[27] K. Willcox, J. Peraire. Balanced model reduction via the proper orthogonal decomposition. AIAA J. 40(11) 2323-2330 (2002).

[28] C.W. Rowley. Model reduction for fluids, using blanaced proper orthogonal decomposition. Int. J. Bifurcation and Chaos 15(3) 997-1013 (2005).

[29] M. Ilak, C.W. Rowley. Reduced-order modeling of channel flow using traveling POD and balanced POD. 3rd AIAA Flow Control Conference, AIAA Paper 2006-3194 (2006).

[30] Z. Bai. Krylov subspace techniques for reduced-order modeling of large-scale dynamical systems. Appl. Numer. Math. 43 9-44 (2002).

[31] Z. Bai, P.M. Dewilde, R.W. Freund. Reduced order modeling, in W.H.A. Schilders, E.J.W. ter Maten (Eds.), Numerical Handbook of Numerical Analysis, Vol. VIII, Elsevier, Amsterdam, pp. 825-895 (2005).

[32] F. Kwasniok. Empirical low-order models of barotropic flow. J. Atmos. Sci. 61(2) 235245 (2004).

[33] R. Everson. L. Sirovich. Karhunen-Loeve procedure for gappy data. J. Optical Society of America A 12(8) 1657-1664 (1995).

[34] S.K. Tiwary, R.A. Rutenbar. Faster, parametric trajectory-based macromodels via localized linear reductions. ICAAD 876-883 (2006). 
[35] K. Carlberg, J. Cortial, D. Amsallem, M. Zahr, C. Farhat. The GNAT non-linear model reduction method and its application to fluid dynamics problems, 6th AIAA Theoretical Fluid Mechanics Conference, AIAA Paper 2011-3112 (2011).

[36] N.C. Nguyen, J. Peraire. An efficient reduced-order modeling approach for non-linear parametrized partial differential equations. Int. J. Numer. Meth. Engng. 76 27-55 (2008).

[37] B. Gustafsson. High Order Difference Methods for Time Dependent PDE, Leipzig, Germany: Springer-Verlag, 2008.

[38] C.W. Rowley, T. Colonius, A.J. Basu. On self-sustained oscillations in two-dimensional compressible flow over rectangular cavities. J. Fluid Mech. 455 315-346 (2002).

[39] M.A. Heroux, R.A. Bartlett, V.E. Howle, R.J. Hoekstra, J.J. Hu, T.G. Kolda, R.B. Lehoucq, K.R. Long, R.P. Pawlowski, E.T. Phipps, A.G. Salinger, H.K. Thornquist, R.S. Tuminaro, J.M. Willenbring, A. Williams, K.S. Stanley. An overview of the trilinos project. ACM Trans. Math. Softw. 31(3) (2005).

[40] B. Kirk, J.W. Peterson, R.H. Stogner, G.F. Carey. libMesh: A C++ library for parallel adaptive mesh refinement/coarsening simulations. Eng. Comput. 22(34) 237-254 (2006).

[41] V. Sankaran, S. Menon. LES of Scalar Mixing in Supersonic Shear Layers, Proceedings of the Combustion Institute, 30(2) 2835-2842 (2004).

[42] L.S. Ukeiley, C. Kannepalli, S. Arunajatesan. Development of Low Dimensional Models for Control of Compressible Flow. Proceedings of the 41st IEEE Conference on Decision and Control, Las Vegas, NV (2002).

[43] F. Genin, S. Menon. Studies of Shock/Turbulent Shear Layer Interaction Using LargeEddy Simulation. Computers and Fluids, 39 800-819 (2010).

[44] F. Genin, S. Menon. Dynamics of Sonic Jet Injection into Supersonic Crossflow. J. Turbulence 11(4) 1-30 (2010). 